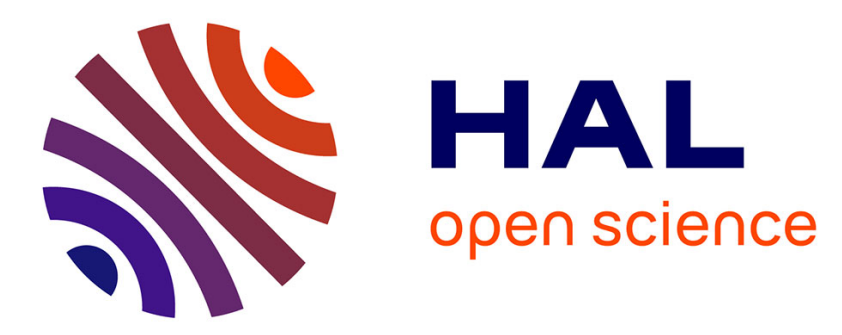

\title{
Wave reflection at a free interface in an anisotropic pyroelectric medium with nonclassical thermoelasticity
}

Abo-El-Nour Abd-Alla, Ivan Giorgio, Luca Galantucci, Abdelmonam M. Hamdan, Dionisio del Vescovo

\section{- To cite this version:}

Abo-El-Nour Abd-Alla, Ivan Giorgio, Luca Galantucci, Abdelmonam M. Hamdan, Dionisio del Vescovo. Wave reflection at a free interface in an anisotropic pyroelectric medium with nonclassical thermoelasticity. Continuum Mechanics and Thermodynamics, 2016, 28 (1-2), pp.67-84. 10.1007/s00161-014-0400-7 . hal-01094877

\section{HAL Id: hal-01094877 \\ https://hal.science/hal-01094877}

Submitted on 14 Dec 2014

HAL is a multi-disciplinary open access archive for the deposit and dissemination of scientific research documents, whether they are published or not. The documents may come from teaching and research institutions in France or abroad, or from public or private research centers.
L'archive ouverte pluridisciplinaire HAL, est destinée au dépôt et à la diffusion de documents scientifiques de niveau recherche, publiés ou non, émanant des établissements d'enseignement et de recherche français ou étrangers, des laboratoires publics ou privés. 


\title{
Wave reflection at a free interface in an anisotropic pyroelectric medium with nonclassical thermoelasticity
}

\author{
Abo-el-nour Abd-alla • Ivan Giorgio • Luca Galantucci • \\ Abdelmonam M. Hamdan • Dionisio Del Vescovo
}

Received: date / Accepted: date

\begin{abstract}
In this paper, the well-established two-dimensional mathematical model for linear pyroelectric materials is employed to investigate the reflection of waves at the boundary between a vacuum and an elastic, transversely isotropic, pyroelectric material. A comparative study between the solutions of (a) classical thermoelasticity, (b) Cattaneo-Lord-Shulman theory and (c) Green-Lindsay theory equations, characterised by none, one and two relaxation times respectively, is presented. Suitable boundary conditions are considered in order to determine the reflection coefficients when incident elasto-electro-thermal waves impinge the free interface. It is established that, in the quasi-electrostatic approximation, three different classes of waves: (i) two principally elastic waves, namely, a quasi-longitudinal Primary $(q P)$ wave and a quasi-transverse Secondary $(q S)$ wave; and (ii) a mainly thermal $(q T)$ wave. The observed electrical effects are, on the other hand, a direct consequence of mechanical and thermal phenomena due to pyroelectric coupling. The computed reflection coefficients of plane $q P$ waves are found to depend upon the angle of incidence, the elastic, electric and thermal parameters of the medium, as well as the thermal relaxation times. The special cases of normal and grazing incidence are also derived and discussed. Finally, the reflection coefficients are computed for Cadmium selenide $(C d S e)$ observing the influence of $(i)$ the anisotropy of the material, (ii) the electrical potential and (iii) temperature variations and (iv) the thermal relaxation times on the reflection coefficients.
\end{abstract}

Keywords Piezoelectricity · Pyroelectricity - Thermal relaxation times - Reflection coefficients · homogeneous waves

A.N. Abd-alla

Department of Mathematics, Faculty of Science, Jazan University, Jazan, Saudi Arabia

E-mail: aboelnourabdalla@yahoo.com

I. Giorgio

Dipartimento di Ingegneria Meccanica e Aerospaziale, Sapienza Università di Roma, Italy

Int. Research Center for the Mathematics and Mechanics of Complex Systems MeMoCS, Italy

E-mail: ivan.giorgio@uniroma1.it

L. Galantucci

Int. Research Center for the Mathematics and Mechanics of Complex Systems MeMoCS, Italy

A. M. Hamdan

Department of Mathematics, Faculty of Science, Sohag University, Sohag, Egypt

D. Del Vescovo

Dipartimento di Ingegneria Meccanica e Aerospaziale, Sapienza Università di Roma, Italy

Int. Research Center for the Mathematics and Mechanics of Complex Systems MeMoCS, Italy 


\section{Introduction}

In this paper, the reflection of waves at the boundary between a vacuum and a half-space filled with an elastic, transversely isotropic, pyroelectric material is studied, under the hypothesis of thermal relaxation times. An analysis of the reflection coefficients for the generated plane waves in dependence of the angle of incidence, the electric-thermal-elastic parameters of the medium and the thermal relaxation times is performed. Finally, a numerical case, referring to the thermo-piezoelectric cadmium selenide $(C d S e)$ material is thus presented.

Studies on wave propagation, reflection and refraction at interfaces in elastic media have always been of great interest and have manifold applications in several fields. For instance, elastic waves propagating through the Earth, i.e. seismic waves, travel through different layers and interfaces: their velocities are influenced by the properties of each layer and whenever these waves face discontinuities in elastic media or between an elastic medium and vacuum, reflection and refraction phenomena occur and need hence to be accurately investigated. Additionally, the properties of reflected and refracted waves are helpful in providing information about the Earth's internal structure and in exploration campaigns for the detection of valuable materials such as oil, minerals, crystals and metals. Evaluation of mineral resources through wave analysis is one of the most attractive technique in terms of costs and time. The study of elastic wave propagation, reflection and refraction is thus of great importance in geophysics, mining and drilling. Since 1970, composites with piezoelectric and piezo-magnetic materials have attracted a significant interest due to their novel magneto-electric effects $[68,25]$. For instance, piezoelectric materials are often employed as resonators with strictly controlled frequencies. Furthermore, piezoelectric ceramics and composites have been extensively employed in many engineering applications, e.g. sensors, actuators and intelligent structures [44]. The mechanics of these so-called smart materials has attracted a considerable academic attention $[62,63$, $64,35,36]$, due to novelty and conspicuous potentialities. As a consequence, in the last decade, many studies on modelling the effective material constants of magneto-electric composites and on their experimental manufacturing have been published $[1,2,26,43,55,64]$.

The response characteristics of piezoelectric structures in an temperature varying environment may change considerably due to pyroelectric effects owned by most piezoelectric materials [54]. Therefore, a thorough understanding of the thermo-piezoelastic (pyroelectric) behaviour is required in order to conceive practical uses of intelligent piezoelectric structures. The thermo-piezoelastic response of these composite materials entails an interaction among mechanical, thermal and electrical properties. The comprehension of the coupling between the thermoelastic and piezoelectric effects implies the quantification of the effects of heat dissipation on wave propagation, at both low and high frequency ranges. Important applications of pyroelectric materials consist in detecting the response of a structure by measuring the induced electric charges (sensing) and/or in reducing excessive responses by imposing an external electric field or a thermal load (actuating). If sensing and actuating are smartly integrated, a so-called intelligent structure can be potentially designed $[8,19,69]$.

In classical theory, thermal disturbances propagate through a continuum with infinite speed. However, few paradoxes are introduced by this assumption, especially for circumstances involving extremely short transient or for temperatures close to absolute zero. For this reason, modified theories have been proposed to account for finite speeds of thermal propagation. The authors clarify that the use of the expression nonclassical dynamical thermoelasticity means a hyperbolic thermoelasticity in which thermal disturbances propagate with finite speeds. Some approaches are based on the general notion of relaxing the heat flux in the classical Fourier heat conduction equation, thereby, introducing a non-Fourier effect. In this context, Lord and Shulman [59], proposed to replace the Fourier law of heat conduction with the Cattaneo law in order to obtain a hyperbolic heat transport equation involving a finite, yet high, speed for thermal signals. On the other hand, Green and Lindsay [24], by including the temperature rate among the constitutive variables, developed a temperature-rate dependent thermoelastic model not violating the Fourier law for bodies having a centre of symmetry. Nonclassical thermoelasticity, piezoelectricity and thermoelastic and piezoelectric coupling, generally for anisotropic elastic solids, have been extensively investigated in a copious number of studies, $[6,9,41,44,54,70,71,72,74]$. On the other hand, only a limited number of analytical and experimental publications on linear piezo-viscoelastic materials, i.e. with dissipation phenomena, may be 
found in the literature. However, in the authors' opinion, dissipation should be taken into account and, for this purpose, the analysis presented in [12] is to be considered.

In particular, fundamental for the present work is the paper by Keith and Crampin [30]. The latter evaluated the energy distribution among waves generated by a plane wave impinging on a boundary between anisotropic media; furthermore, a comprehensive account is presented for longitudinal Primary $(P)$, transverse $S$ econdary $(S V$ and $S H$ ) waves at a boundary between an isotropic half-space and an orthorhombic olivine half-space. On the other hand, another key study is [31] where Kuang and Yuan, applying the Lord and Shulman model, investigated and simulated with numerical examples two-dimensional wave reflections at the free interface of a semi-infinite pyroelectric medium. In the framework of non classical thermoelasticity theory, further contributions were performed by Sharma et al. [56,57] who discussed the reflection of quasi- $P(q P)$, quasi- $S(q S)$ and thermal waves from a transversely isotropic, pyroelectric half-space with $a$ ) stress-free, thermally insulated and isothermal open circuit boundaries [56] and $b$ ) charge- and stress-free boundaries [57]. Waves reflection from pre-stressed boundaries of transversely isotropic, pyroelectric halfspace were analyzed by Singh [61], who also investigated the plane wave velocities with both Lord-Shulman and Green-Lindsay models [60]. Very recently, results related to the topics addressed in the present paper were reported in the original investigations by Yuan et al. [75] and Abd-alla [3,4,5], where wave refraction and reflection in anisotropic piezoelectric media are studied. For the sake of completeness, it is worth citing some other relevant papers: a) the results obtained by Tomar and Khurana [67], concerning the reflection of plane waves from a stress-free plane boundary of an electro-micro elastic solid, b) the study by Kumar and Rupender [32], on electro-microstretched generalised thermoelastic solids c) and the articles by Kumar and Kumar [33] and Singh [62] on thermoelastic media with voids. In this last context, several studies are relevant to the topics under investigation: Quiligotti et al. [50] inspected particular generalised continua constituted by porous deformable solids infused with an inviscid compressible fluid; Berezovski and Maugin [10] considered the propagation of both thermoelastic waves and phase-transition fronts in a unified framework, based on thermodynamic consistency conditions; Placidi et al. $[46,49]$ studied the propagation of bulk transverse and longitudinal waves and the influence of pre-stresses, deriving evolution equations through a variational approach [49]; Madeo et al. [16,37,38], starting from an extended Hamilton-Rayleigh principle, defined a general set of boundary conditions related to fluid-permeable interfaces between dissimilar fluid-filled porous matrices and the effects of confined fluid streams. Many investigations deal with interfaces endowed with mass, deformation energy, frictional or other physical properties: e.g. Misra et al. [39,40] suggested how micro-mechanical, frictional effects or interfacial roughness and nonlinearities could be incorporated in the models; dell'Isola et al. [17] studied reflection and transmission of plane waves at flat displacement discontinuity surfaces, for a specific class of second gradient continua $[18,65]$. Concerning this last class of materials, it is possible to observe wave trapping phenomena [53] and reflection and transmission of compression [52] and shear [48] waves at planar interfaces formed by porous matrix discontinuities. Other relevant studies take into account micromorphic models $[21,34,42]$, dissipative contact phenomena $[7,13]$ and stability and bifurcation of piezoelectric structures [29,28].

The organisation of the present paper is as follows. In Section 2 the mathematical formulation employed for the description of pyroelectric media is outlined, with particular attention on the differentiation deriving from the three thermal models adopted. In Section 2.2, the mathematical expression of the waves are shown and the dispersion relation is derived. In Section 3.1 a comparison among different model solutions is exposed. The reflection coefficients are deduced in Section 3.2 and, finally, the conclusions and the discussion of the results are summarised in Section 4.

Notation: subscripted commas indicate spatial derivatives, while superimposed dots denote time derivatives.

\section{Model of pyroelectric media}

We consider an infinite, homogeneous, transversely isotropic, pyroelectric medium, initially at uniform temperature $T_{0}$ and electrical potential $\varphi_{0}$, occupying a semi-infinite space adjacent to a vacuum half- 


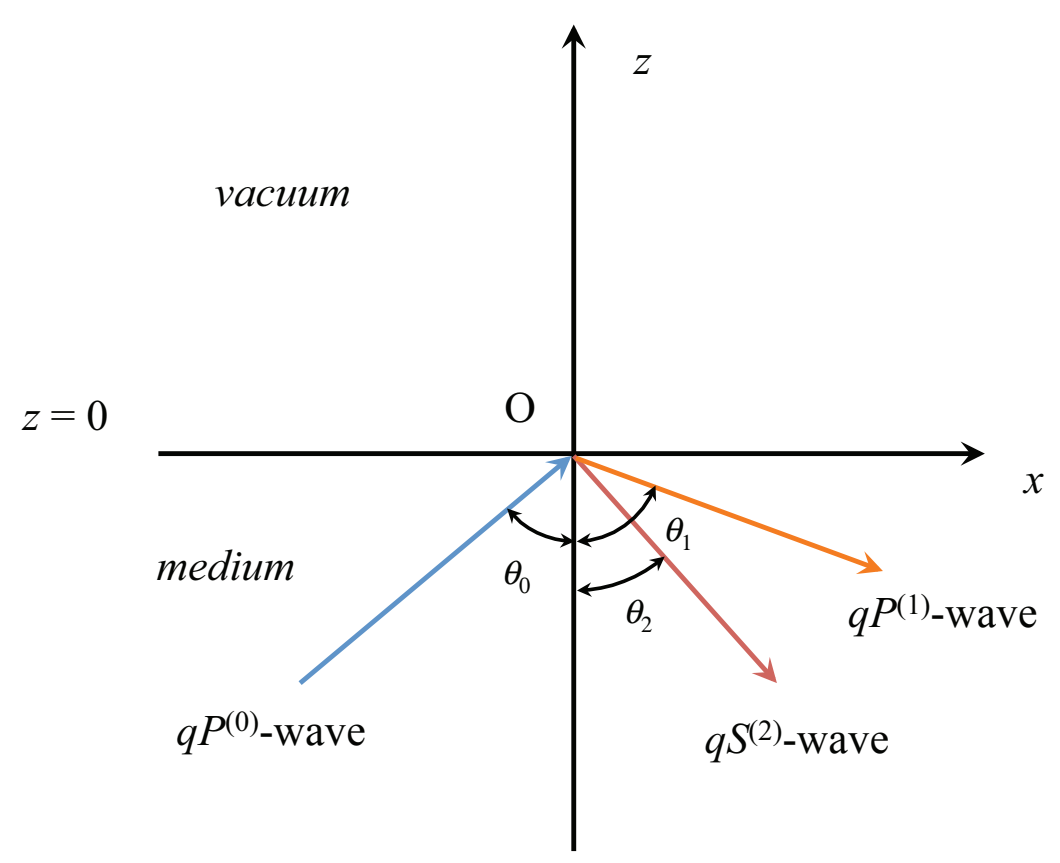

Fig. 1 Geometry of incident and reflected waves.

space. The coordinate system $(O, x, y, z)$ is chosen such that the direction of wave propagation is parallel to the $x z$-plain so that all particles on a line parallel to the $y$-axis are equally displaced. Therefore, all the fields are independent of the $y$-coordinate: the displacement vector $\boldsymbol{u}=\left(u_{1}, 0, u_{3}\right)$, the electrical potential $\varphi(x, z, t)$ and the temperature change $\vartheta=T-T_{0}$, where $T$ is the absolute temperature of a point $\boldsymbol{x}=$ $\left(x_{1}, 0, x_{3}\right)=(x, 0, z)$ of the medium. We define the $z$-axis to be along the axis of symmetry of the material, determined by the elastic constants and the thermal and electrical poling. Specifically, the region $z \leqslant 0$ locates the medium and the region $z>0$ the vacuum. It is assumed that the interface between the two regions, $z=0$, is stress-free, electrically shorted and isothermic.

In order to consider a linear model, we assume small strains $\varepsilon_{k l}=\left(u_{k, l}+u_{l, k}\right) / 2$ and small temperature changes $\left|\vartheta / T_{0}\right| \ll 1$. We assume the quasi-electrostatic approximation instead of the full electromagnetic equations because the phase velocities of elastic waves are five orders of magnitude less than the velocities of electromagnetic waves, thus we suppose the electric vector field $E$ is derivable from a potential, i.e. $E_{k}=-\varphi, k$. The pyroelectric medium is described by the mechanical, electric and thermal fields. The constitutive equations for the Piola stress tensor $\sigma$, for the electric displacement field $\boldsymbol{D}$ and for the entropy density $S$ can be written in component form as follows:

$$
\left\{\begin{array}{l}
\sigma_{i j}=c_{i j k l} \varepsilon_{k l}-e_{k i j} E_{k}-\gamma_{i j}\left(\vartheta+\delta_{G L} t_{1} \dot{\vartheta}\right) \\
D_{k}=e_{k i j} \varepsilon_{i j}+p_{i k} E_{i}+d_{k}\left(\vartheta+\delta_{G L} t_{1} \dot{\vartheta}\right) \\
\rho S=\gamma_{i j} \varepsilon_{i j}+d_{k} E_{k}+\frac{\rho C_{\varepsilon}}{T_{0}} \vartheta+\left(\delta_{L S}+\delta_{G L}\right) \frac{\rho C_{\varepsilon}}{T_{0}} t_{0} \dot{\vartheta}+\delta_{G L} \frac{b_{i}}{T_{0}} \vartheta_{, i}
\end{array}\right.
$$

The third equation in Eqs. (1) represents the Rayleigh potential density which accounts for dissipative phenomena. In addition, the heat conduction equation is assumed

$$
q_{i}=-K_{i j} \vartheta_{, j}-\delta_{L S} t_{0} \dot{q}_{i}-\delta_{G L} b_{i} \dot{\vartheta}
$$

In Eqs. (1)-(2) the symbols employed have the following meaning: $c_{i j k l}$ stands for the elastic stiffness tensor, $e_{k i j}$ the piezoelectric tensor; $\gamma_{i j}$ the thermo-mechanical tensor, $p_{i k}$ the dielectric permittivity tensor, $d_{k}$ the 
pyroelectric constants, $\rho$ the mass density of the medium, $C_{\varepsilon}$ is the specific heat capacity at constant strain and constant electric vector field and $K_{i j}$ are the components of the thermal conductivity tensor. The nondimensional constants $\delta_{L S}$ and $\delta_{G L}$ allow to switch from three different theories: the Classical Dynamical coupled theory of thermoelasticity, referred to as CD-model, and two generalisations, the Lord-Shulman theory, referred to as LS-model, characterised by one relaxation time [59] and the Green-Lindsay theory, referred to as GL-model, characterised by two relaxation times [24]. The relaxation time $t_{0}$ is the timelag needed before a steady-state heat conduction is reached when a temperature gradient is suddenly imposed [59]. The relaxation time $t_{1}$ is a characteristic parameter of the Green-Lindsay theory.

Hence, we obtain three different models, respectively named:

1. CD-model, with $\delta_{L S}=\delta_{G L}=0$;

2. LS-model, with $\delta_{L S}=1, \delta_{G L}=0$;

3. GL-model, with $\delta_{L S}=0, \delta_{G L}=1$.

Let us observe that in Eq. (2), LS-model employs the Maxwell-Cattaneo model of heat conduction instead of the classical Fourier relation, while GL-model includes also $\dot{\vartheta}$ in the list of independent constitutive variables.

The elastic stiffness tensor, $c_{i j k l},[66,27]$, can be written as

$$
c_{i j k l}=\sum_{r=1}^{6} c_{r} I_{i j k l}^{(r)}
$$

where, in components, the fourth-order tensors $I_{i j k l}^{(r)}$ are

$$
\begin{aligned}
I_{i j k l}^{(1)} & =\frac{1}{2}\left(\delta_{i k} \delta_{j l}+\delta_{i l} \delta_{i k}\right), & I_{i j k l}^{(2)} & =\delta_{i j} \delta_{k l}, \\
I_{i j k l}^{(3)} & =N_{i j} \delta_{k l}, & I_{i j k l}^{(4)} & =\delta_{i j} N_{k l}, \\
I_{i j k l}^{(5)} & =\frac{1}{2}\left(\delta_{i k} N_{j l}+\delta_{i l} N_{i k}+\delta_{j l} N_{i k}+\delta_{j k} N_{i l}\right), & I_{i j k l}^{(6)} & =N_{i j} N_{k l},
\end{aligned}
$$

being $c_{r}$ the elastic material parameters, the tensor $N_{i j}=n_{i} n_{j}$ and $n_{i}$ the components of the unit vector parallel to the axis of transverse isotropy and the tensor $\delta_{i j}$ is the Kronecker delta.

The conservation of linear momentum, the conservation of charge and the entropy balance equation can be expressed as:

$$
\left\{\begin{array}{l}
\sigma_{i k, k}=\rho \ddot{u}_{i} \\
D_{k, k}=0 \\
\rho T_{0} \dot{S}=-q_{i, i}
\end{array}\right.
$$

Inserting the constitutive Eqs. (1) into Eqs. (5), the thermo-kinetic model for a linear pyroelectric medium can be obtained $[59,24,58]$ :

$$
\left\{\begin{array}{l}
c_{i j k l} \varepsilon_{k l, j}+e_{k i j} \varphi, k j-\gamma_{i k}\left(\vartheta+\delta_{G L} t_{1} \dot{\theta}\right)_{, k}=\rho \ddot{u}_{i} \\
e_{k i j} \varepsilon_{i j, k}-p_{i k} \varphi_{, i k}+d_{k}\left(\vartheta+\delta_{G L} t_{1} \dot{\vartheta}\right)_{, k}=0 \\
K_{i j} \vartheta_{, i j}=\rho C_{\varepsilon}\left(\dot{\vartheta}+\left(\delta_{L S}+\delta_{G L}\right) t_{0} \ddot{\vartheta}\right)+T_{0}\left[\gamma_{i j}\left(\dot{\varepsilon}_{i j}+\delta_{L S} t_{0} \ddot{\varepsilon}_{i j}\right)+d_{k}\left(\dot{E}_{k}+\delta_{L S} t_{0} \ddot{E}_{k}\right)\right]-\delta_{G L} 2 \frac{b_{i}}{T_{0}} \dot{\vartheta}_{, i}
\end{array}\right.
$$

We pose $b_{i}$ equal to zero, the material having a centre of symmetry. The last equation of (6) for the CD-model is a parabolic equation and consequently leads to the nonphysical behaviour of infinite velocity for the propagation of thermal disturbances; for the LS-model and the GL-model, this last equation turns hyperbolic and, in the uncoupled case and for isotropic diffusion tensor $K_{i j}=K \delta_{i j}$, it results in a finite characteristic velocity, i.e. $v_{c}=\sqrt{K /\left(\rho C_{\varepsilon} t_{0}\right)}$. Hence, in these hyperbolic cases, the perturbations $\vartheta$ are 
dissipative wavelike solutions, in the sense that no information can propagate with speed higher than a definite limit, see e.g. fig. 11.

Finally, it is worth mentioning that the pyroelectric medium can be treated as a metamaterial with a micro-structure whose behaviour is based on a multi-field coupling [20].

\subsection{Boundary conditions}

The boundary conditions at the surface $z=0$ are the following, with superscripts $(n)$ indicating the incident $(0)$ or the reflected $(1,2)$ waves (figure 1 ):

Mechanical conditions: the surface of the half space is assumed to be stress-free

$$
\sum_{n=0,1,2} \sigma_{33}^{(n)}=0, \quad \sum_{n=0,1,2} \sigma_{13}^{(n)}=0
$$

Electrical conditions: the surface of the half-space is assumed to be electrically grounded

$$
\sum_{n=0,1,2} \varphi^{(n)}=0
$$

Thermal conditions: the interface with vacuum is assumed to be an isothermal surface with temperature $T_{0}$ :

$$
\sum_{n=0,1,2} \vartheta^{(n)}=0
$$

2.2 Plane waves in pyroelectric media

The solutions of Eqs. (6) are plane waves travelling in the $\boldsymbol{n}$ direction that can be represented by wavefunctions

$$
\left\{\begin{array}{l}
u_{\alpha}(\boldsymbol{x}, t)=A_{\alpha} e^{i(-k \boldsymbol{n} \cdot \boldsymbol{x}+\omega t)} \\
\varphi(\boldsymbol{x}, t)=B e^{i(-k \boldsymbol{n} \cdot \boldsymbol{x}+\omega t)} \\
\vartheta(\boldsymbol{x}, t)=C e^{i(-k \boldsymbol{n} \cdot \boldsymbol{x}+\omega t)}
\end{array}\right.
$$

where $A_{\alpha}$ is the amplitude of the displacement wave, $B$ is the amplitude of the electrical potential wave, $C$ is the amplitude of the thermal wave, i.e. unknown amplitudes to be determined by means of the boundary conditions; $k$ is the wave number, $\omega$ is the wave angular frequency and $\boldsymbol{x}$ is an arbitrary point on the $x z$-plane. We remark that the wave number $k$ is complex due to the thermal dissipation introduced by the third of Eqs. (6), and then we can assume

$$
k=k^{(\Re)}+i k^{(\Im)}
$$

Herein, we assume that the propagation $\left(k^{(\Re)} \boldsymbol{n}\right)$ and attenuation $\left(k^{(\Im)} \boldsymbol{n}\right)$ directions coincide, under the homogeneous wave condition, while in a more general inhomogeneous case, these two vectors are not collinear [73]. Rewriting the Eq. (10), we obtain a wave solution in the form

$$
\left\{\begin{array}{l}
u_{\alpha}(\boldsymbol{x}, t)=A_{\alpha} e^{k^{(\Im)} n \cdot \boldsymbol{x}} e^{i\left(-k^{(\Re)} \boldsymbol{n} \cdot \boldsymbol{x}+\omega t\right)} \\
\varphi(\boldsymbol{x}, t)=B e^{k^{(\Im)} \boldsymbol{n} \cdot \boldsymbol{x}} e^{i\left(-k^{(\Re)} \boldsymbol{n} \cdot \boldsymbol{x}+\omega t\right)} \\
\vartheta(\boldsymbol{x}, t)=C e^{k^{(\Im)} \boldsymbol{n} \cdot \boldsymbol{x}} e^{i\left(-k^{(\Re)} \boldsymbol{n} \cdot \boldsymbol{x}+\omega t\right)}
\end{array}\right.
$$

The phase velocity of this plane wave is directed as $\boldsymbol{n}$ with amplitude 


$$
v=\frac{\omega}{k^{(\Re)}}
$$

and its attenuation is due to the imaginary part $k^{(\Im)}$.

Substituting Eqs. (10) into governing Eqs. (6), dropping the common exponential factor, we obtain a linear algebraic system of four equations with four unknowns, i.e. the amplitudes $A_{1}, A_{3}, B, C$. This set of equations displays nontrivial solutions if the determinant of the characteristic matrix $\Lambda$ is zero

$$
\operatorname{det}[\Lambda(k, \omega)]=0
$$

which represents the dispersion relation for the pyroelectric medium, resulting in an eighth order polynomial in the wavenumber $k$, as a function of the angular frequency $\omega$.

\section{Numerical simulation and discussion}

To perform a numerical analysis of this problem, we consider, as pyroelectric material, Cadmium Selenide $(C d S e)$ which has a hexagonal crystal structure. Its physical properties, taken from references $[9,57,60]$, are listed in Table 1. Specifically the elastic material parameters $c_{r}$ in Eq. (3) are related to the moduli $c_{i j}$ in Voigt notation by the following relations [11,27]

$$
\begin{array}{ll}
c_{1}=c_{11}-c_{12}=2 c_{66}, & c_{2}=c_{12}, \quad c_{3}=c_{4}=c_{13}-c_{12}, \\
c_{5}=2\left(c_{55}-c_{66}\right), & c_{6}=c_{11}+c_{33}-2 c_{13}-4 c_{55},
\end{array}
$$

when the axis of isotropy is along the $z$-direction; analogously, for the piezoelectric constants $e_{i q}$ we can write

$$
\begin{aligned}
& e_{i q}=e_{i k l}, \quad k=l, q=1,2,3 \\
& e_{i q}=2 e_{i k l}, \quad k \neq l, q=4,5,6
\end{aligned}
$$

\subsection{General solution: comparison among the three models}

Eq. (14) gives, for each model considered, four pairs of complex roots. It is important to recall that each of these four pairs leads to a wave solution which is simultaneously composed by three components, i.e. a mechanical, an electrical and a thermal (Eqs. (12)). Due to the quasi-electrostatic approximation, the roots of one of the pairs are null and for the other three waves the electrical component, corresponding to the second expression of Eqs. (12), stems exclusively from the coupling with mechanical and thermal components. The three non-trivial solutions, each characterised by a specific dispersion relation, thereinafter are referred to as:

1. quasi-longitudinal Primary $(q P)$ wave with the mechanical component quasi-longitudinal;

2. quasi-transverse Secondary $(q S)$ wave with the mechanical component quasi-transverse;

3. thermal wave or quasi-Thermal wave $(q T)$, with a dominant thermal component.

For each wave, the phase velocity closely approximates the value of the correspondent uncoupled subsystem; in other words, we assume that the first two solutions are mainly mechanical, while the third is predominantly thermal.

Since the differential Eqs. (6) are dissipative and the medium is anisotropic, the propagation velocity depends on the angle of propagation and is greater for the $q P$ wave than for the $q S$ one.

Figs. 2-10 show the graphs of propagation velocities $v$ and attenuation factors $k^{(\Im)} v s$ propagation angle $\theta$ and angular frequency $\omega$, for $q P, q S$ and $q T$ waves, for the three models.

For the CD-model, Figs. 2 and 3 show for the mechanical waves that the velocity $v$ is substantially independent of the angular frequency $\omega$; concerning the attenuation, the $q P$ solution shows a higher $k^{(\Im)}$ with respect to $q S$ wave; for $q S$ solution, Fig. $3, k^{(\Im)}$ vanishes for propagation angles $\theta$ close to zero or greater 
Table 1 Physical properties of Cadmium Selenide $(C d S e)$

\begin{tabular}{lll}
\hline Symbol & Value & Unit \\
\hline$c_{11}$ & $7.41 \times 10^{10}$ & $\mathrm{Nm}^{-2}$ \\
\hline$c_{12}$ & $4.52 \times 10^{10}$ & $\mathrm{Nm}^{-2}$ \\
\hline$c_{13}$ & $3.93 \times 10^{10}$ & $\mathrm{Nm}^{-2}$ \\
\hline$c_{33}$ & $8.36 \times 10^{10}$ & $\mathrm{Nm}^{-2}$ \\
\hline$c_{44}$ & $1.32 \times 10^{10}$ & $\mathrm{Nm}^{-2}$ \\
\hline$e_{13}$ & -0.160 & $\mathrm{Cm}^{-2}$ \\
\hline$e_{33}$ & 0.374 & $\mathrm{Cm}^{-2}$ \\
\hline$e_{15}$ & -0.138 & $\mathrm{Cm}^{-2}$ \\
\hline$d_{3}$ & $-2.94 \times 10^{-6}$ & $\mathrm{CK}^{-1} \mathrm{~m}^{-2}$ \\
\hline$\gamma_{11}$ & $0.621 \times 10^{6}$ & $\mathrm{NK}^{-1} \mathrm{~m}^{-2}$ \\
\hline$\gamma_{33}$ & $0.551 \times 10^{6}$ & $\mathrm{NK}^{-1} \mathrm{~m}^{-2}$ \\
\hline$p_{11}$ & $8.26 \times 10^{-11}$ & $\mathrm{C}^{2} \mathrm{~N}^{-1} \mathrm{~m}^{-2}$ \\
\hline$p_{33}$ & $9.03 \times 10^{-11}$ & $\mathrm{C}^{2} \mathrm{~N}^{-1} \mathrm{~m}^{-2}$ \\
\hline$K_{11}$ & 9 & $\mathrm{WK}^{-1} \mathrm{~m}^{-1}$ \\
\hline$K_{33}$ & 9 & $\mathrm{WK}^{-1} \mathrm{~m}^{-1}$ \\
\hline$C_{\varepsilon}$ & 260 & $\mathrm{~J} \mathrm{~kg}^{-1} \mathrm{~K}^{-1}$ \\
\hline$\rho$ & 5504 & $\mathrm{~kg} \mathrm{~m}^{-3}$ \\
\hline$T_{0}$ & 298 & $\mathrm{~K}^{-1}$ \\
\hline
\end{tabular}

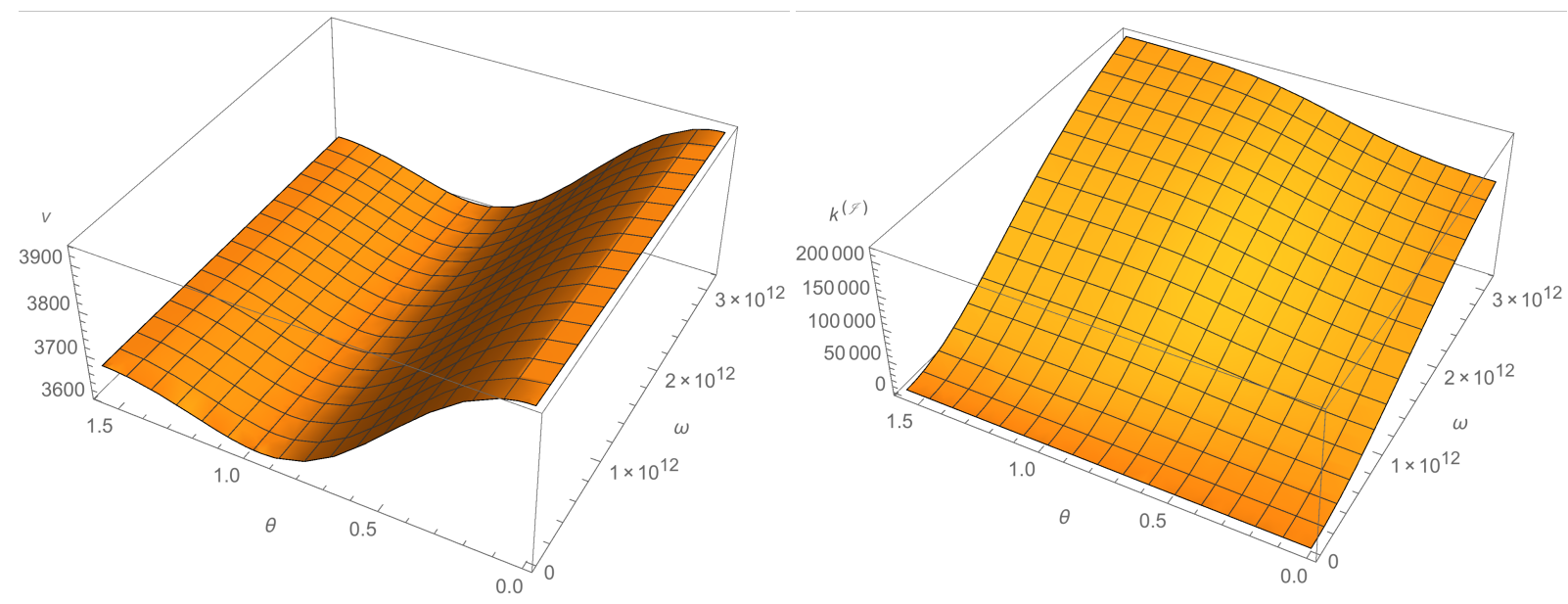

Fig. 2 CD-model, $q P$ wave: phase velocity $v$ and attenuation factor $k^{(\Im)} v s$ the propagation angle $\theta$ and the angular frequency $\omega$. 


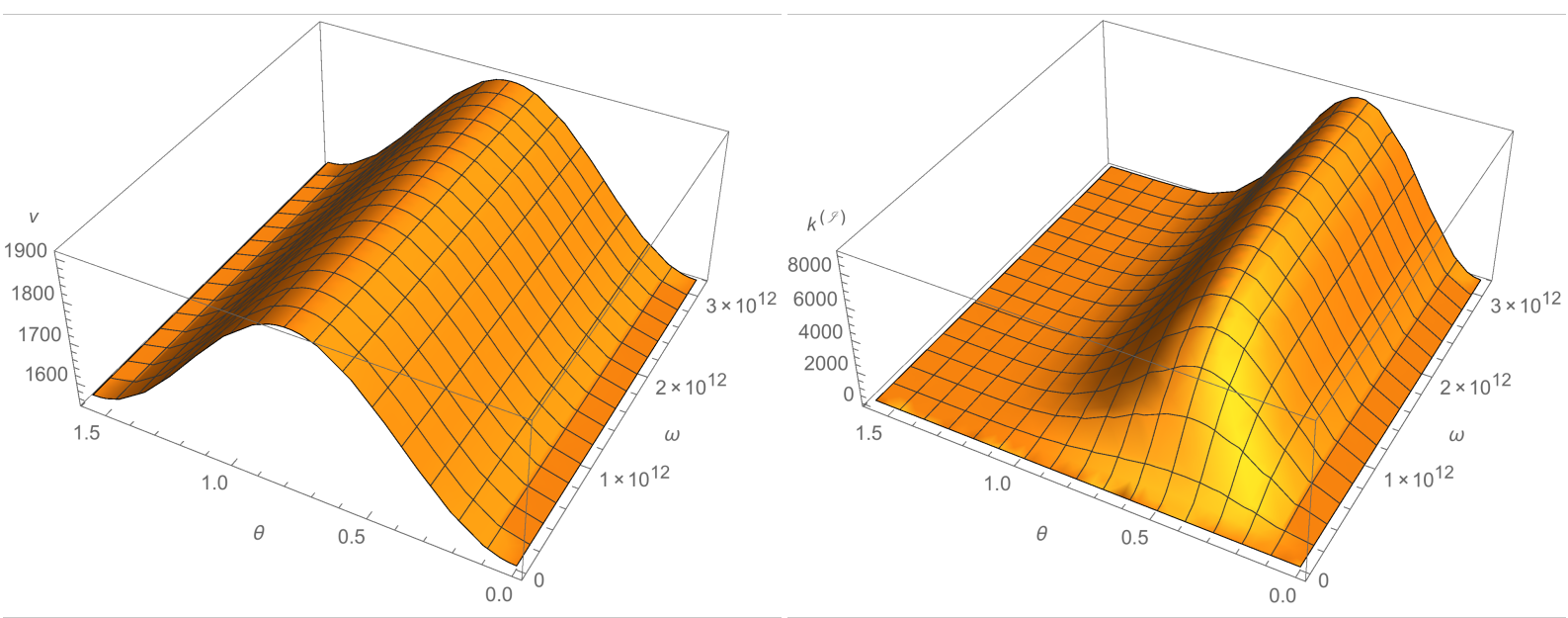

Fig. 3 CD-model, $q S$ wave: phase velocity $v$ and attenuation factor $k^{(\Im)} v s$ the propagation angle $\theta$ and the angular frequency $\omega$.

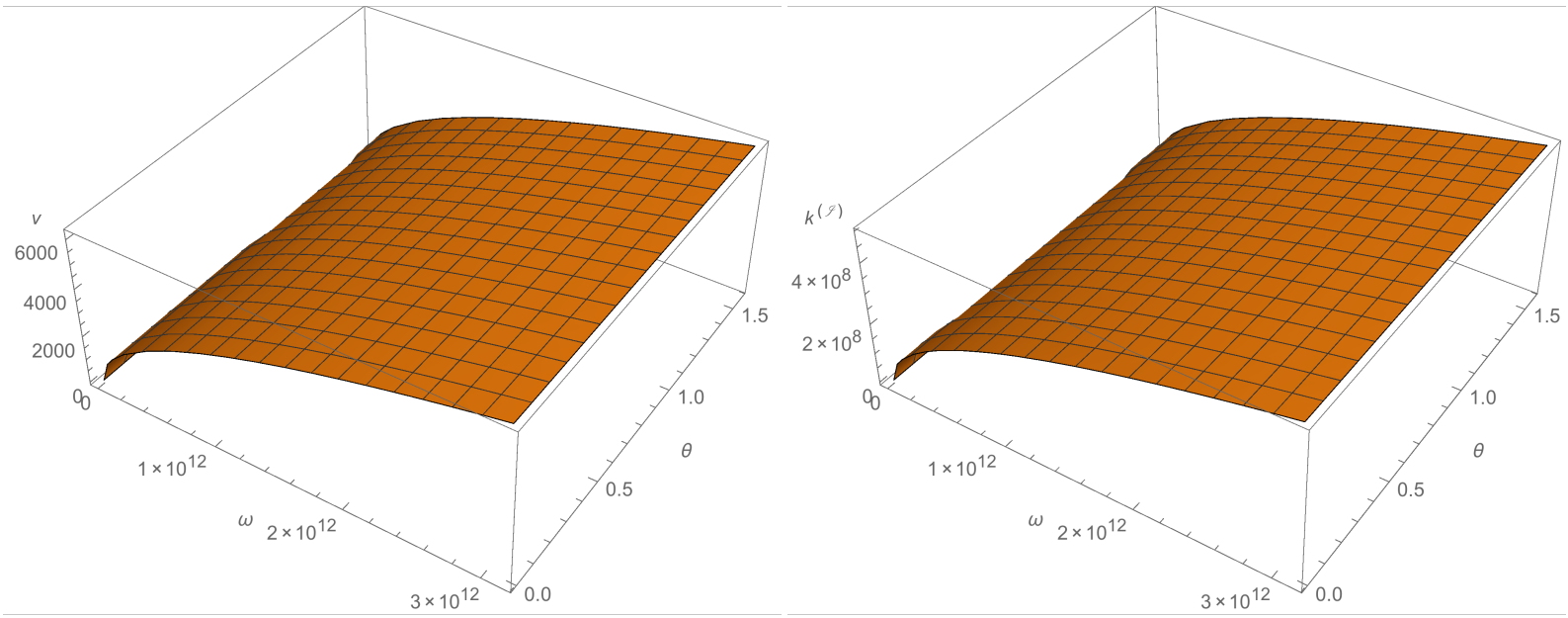

Fig. 4 CD-model, thermal wave: phase velocity $v$ and attenuation factor $k^{(\Im)} v s$ the propagation angle $\theta$ and the angular frequency $\omega$.

than $\pi / 3$. In Fig. 4, the thermal wave manifests the parabolic character of Eq. $(6)_{3}\left(\delta_{L S}=\delta_{G L}=0\right)$, with a propagation velocity $v$ growing indefinitely with increasing angular frequency; similar considerations can be done for the attenuation factor $k^{(\Im)}$.

For the LS-model, Figs. 5, 6 and 7 show the solutions assuming a relaxation time $t_{0}=1 \times 10^{-12} \mathrm{~s}$. The propagation velocities for $q P$ and $q S$ waves do not show significant differences compared to the CD-model; the attenuation factor $k^{(\Im)}$ of the $q P$ wave is lower in LS-model than in CD-model, while the opposite occurs for the $q S$ solution. Concerning the thermal solution, a different behaviour can be observed due to the hyperbolic character of Eq. $(6)_{3}\left(\delta_{L S}=1, \delta_{G L}=0\right)$; in particular, a characteristic upper bound velocity, about $2500 \mathrm{~m} / \mathrm{s}$, is identified, i.e. no wave can propagate with higher velocities.

For the GL-model, Figs. 8, 9 and 10 depict the solutions assuming the two relaxation times $t_{0}=$ $1 \times 10^{-12} \mathrm{~s}$ and $t_{1}=40 t_{0}$. For the $q P$ wave, a slightly dispersive behaviour is observed in this model: the phase velocity $v$ depends on the angular frequency $\omega$ for any angle $\theta$; on the contrary, this effect results 


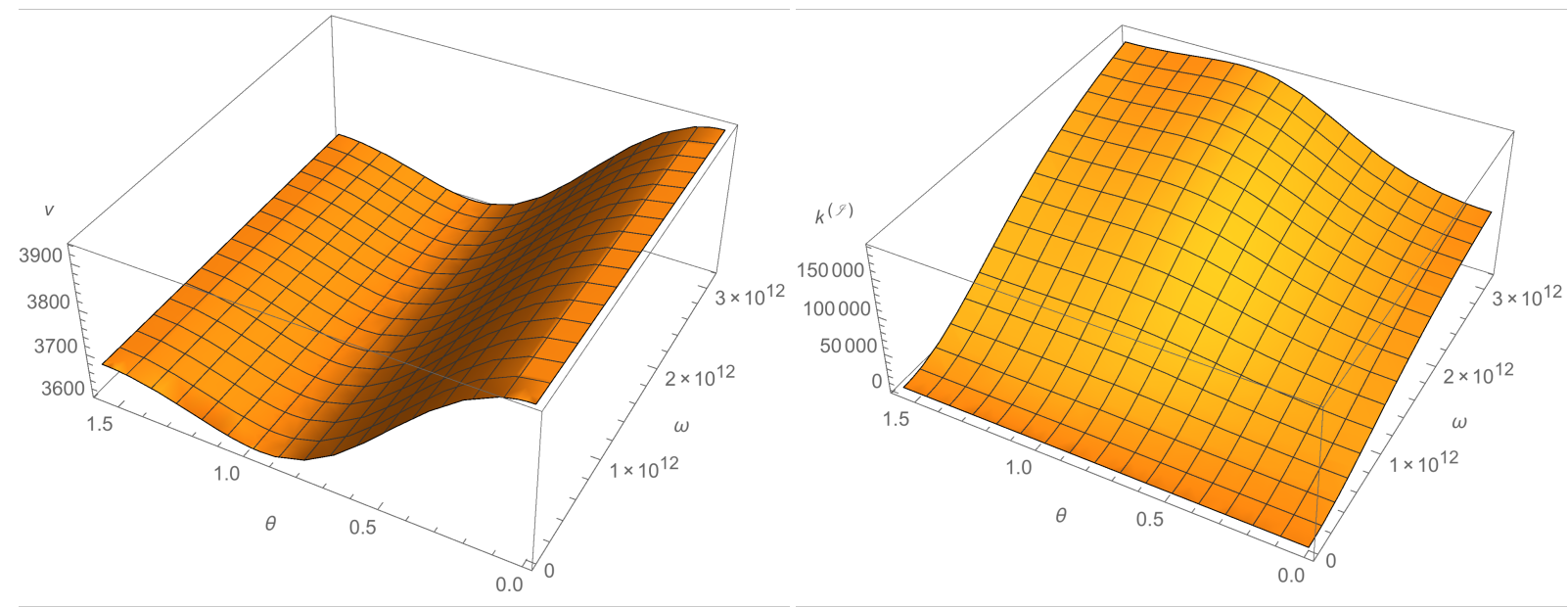

Fig. 5 LS-model, $q P$ wave: phase velocity $v$ and attenuation factor $k^{(\Im)} v s$ the propagation angle $\theta$ and the angular frequency $\omega$. Relaxation time $t_{0}=1 \times 10^{-12} \mathrm{~s}$.

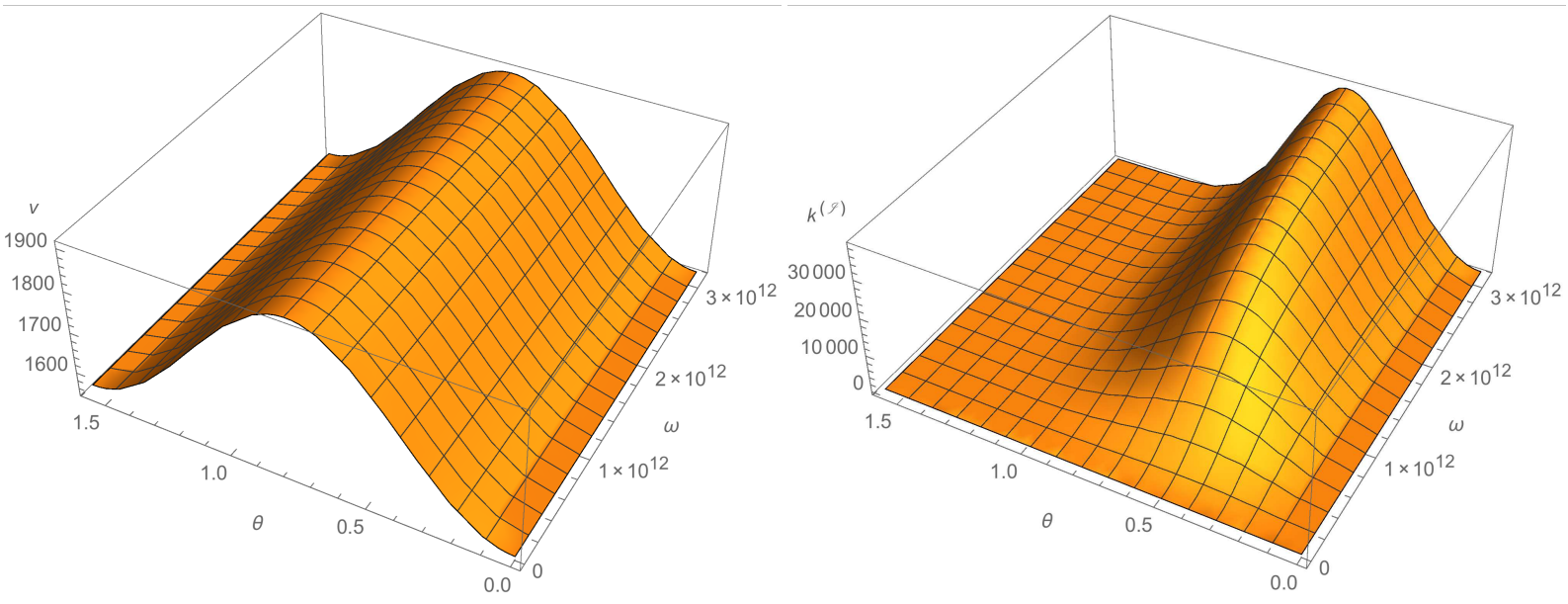

Fig. 6 LS-model, $q S$ wave: phase velocity $v$ and attenuation factor $k^{(\Im)} v s$ the propagation angle $\theta$ and the angular frequency $\omega$. Relaxation time $t_{0}=1 \times 10^{-12} \mathrm{~s}$.

negligible for the $q S$ solution; furthermore, the attenuation factor $k^{(\Im)}$ for $q P$ and $q S$ waves is the highest of the three models. For the thermal wave, a analogous behaviour to the LS-model is found, with a similar limit propagation velocity, due to Eq. $(6)_{3}\left(\delta_{L S}=0, \delta_{G L}=1\right)$ also hyperbolic.

For the thermal waves, the results about the velocity $v$ in Figs. 4, 7 and10 are compared in Fig. 11 where, considering that there is no significant dependence on the propagation angle $\theta$, the velocity $v$ and the attenuation factor $k^{(\Im)}$ are represented $v s \omega$ only. Both LS and GL models clearly show a characteristic velocity, arising from their hyperbolic nature. Moreover, parametric analyses have been performed to investigate the role of relaxation times. Fig. 12 exhibits the phase velocity $v$ for relaxation time $t_{0}$ equal to $1,2,3$ and $4 \mathrm{ps}$ in the case of LS-model and for relaxation time $t_{1}$ equal to $10,400,800,1200 \mathrm{ps}$ for the GL-model with constant $t_{0}=1$ ps. From this figure, it is clear that the increase of both relaxation times implies a decrease of the characteristic velocity of the thermal wave. 


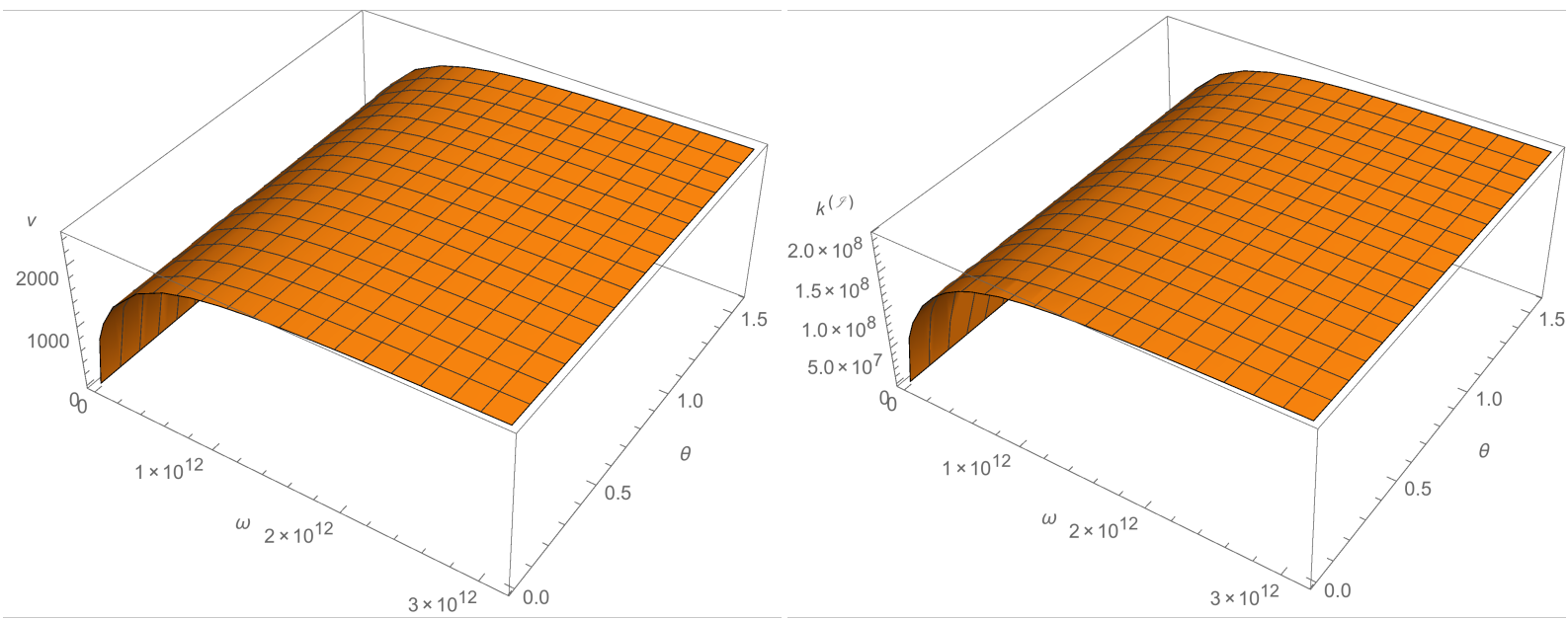

Fig. 7 LS-model, thermal wave: phase velocity $v$ and attenuation factor $k^{(\Im)} v s$ the propagation angle $\theta$ and the angular frequency $\omega$. Relaxation time $t_{0}=1 \times 10^{-12} \mathrm{~s}$.

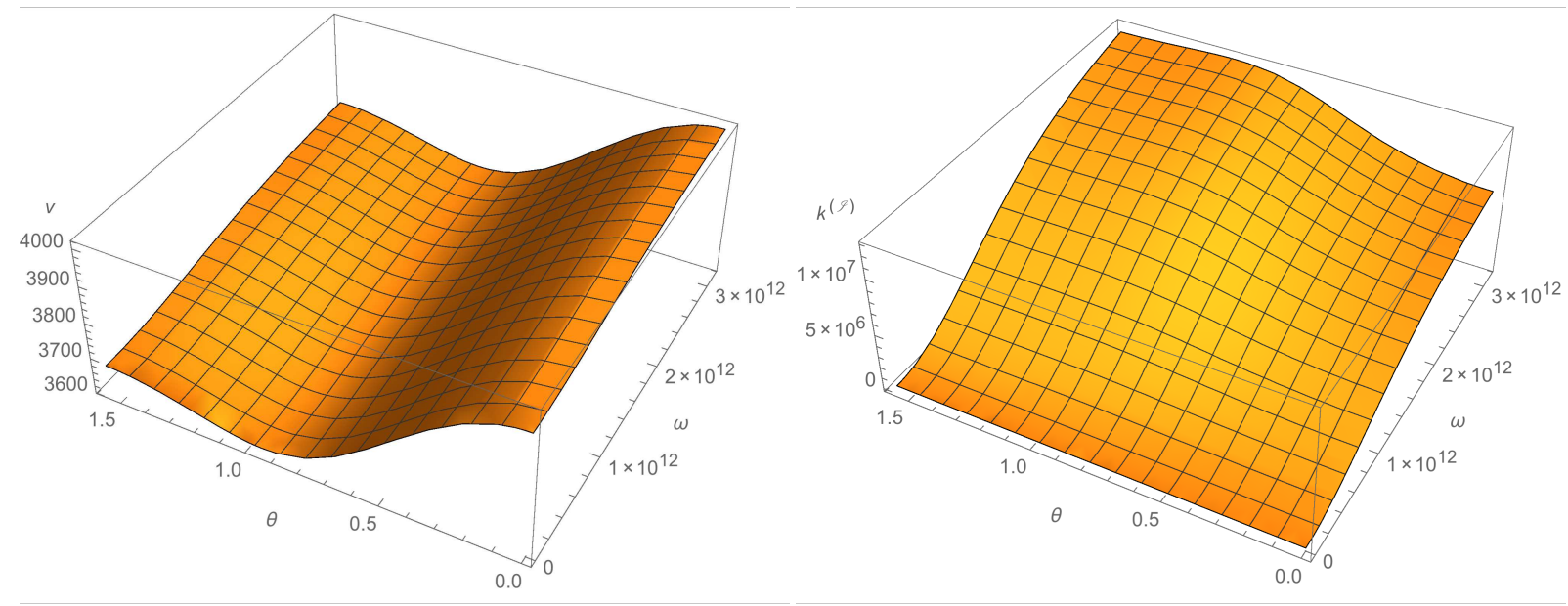

Fig. 8 GL-model, $q P$ wave: phase velocity $v$ and attenuation factor $k^{(\Im)} v s$ the propagation angle $\theta$ and the angular frequency $\omega$. Relaxation times $t_{0}=1 \times 10^{-12} \mathrm{~s}$ and $t_{1}=40 t_{0}$.

It is worth to signalise that the attenuation factor $k^{(\Im)}$ resulted in all cases to have very low importance in the frequency range of interest for mechanical systems.

For $q P$ waves, the mechanical component has a displacement of the medium quasi parallel to the propagation of energy transfer, i.e. the direction of travel of the wave; analogously, for $q S$ waves the displacement of oscillations is quasi perpendicular to the propagation. For the sake of completeness, Fig. 13 shows the angle $\Delta \theta$ between the medium displacement and the travel wave direction $v s \omega$ for any solution of Eq. (6) and for $\theta=\pi / 6$, for which the maximum deviation $\Delta \theta$ occurs. The results reported show that the mechanical components can be effectively considered longitudinal or transverse with a very good approximation for the three models. Finally, only in the $q P$ wave of the GL-model a significant variation of $\Delta \theta$ with the frequency is observed.

The following list of references is very useful in coping with several numerical problems, involving different materials described by more field variables: [22, 23, 15, 14,51]. 


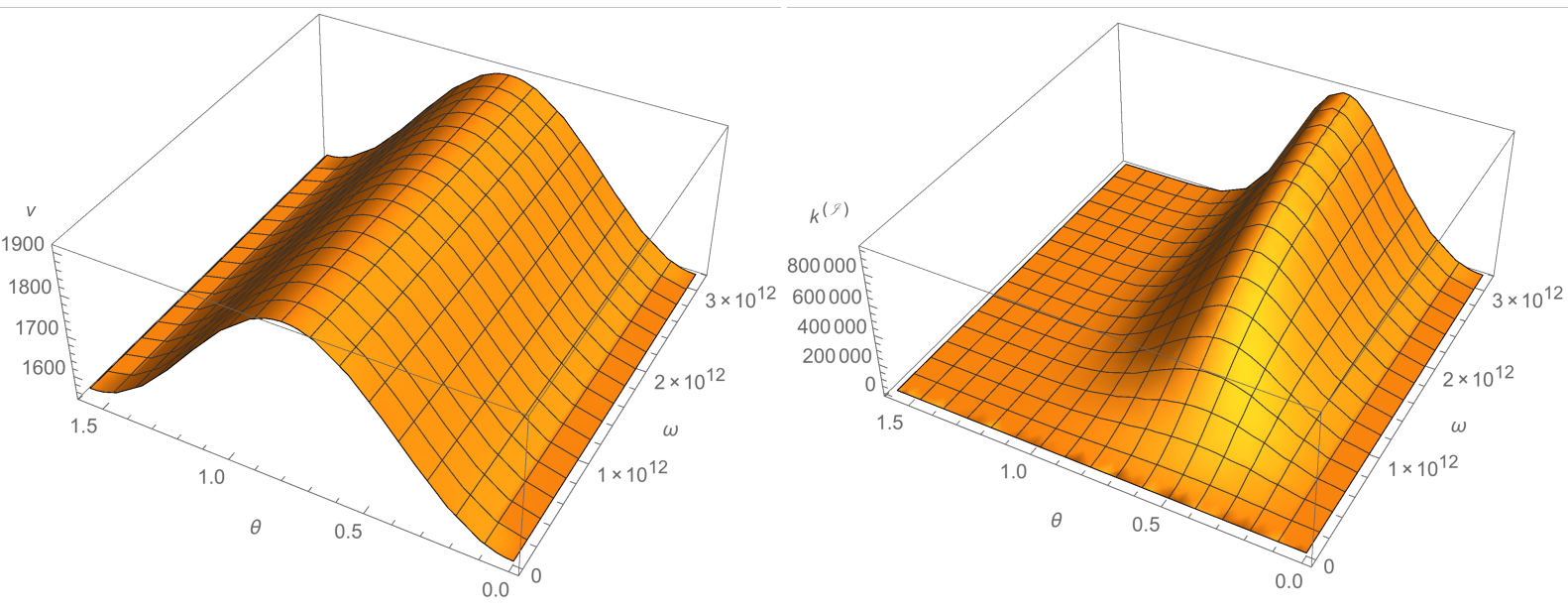

Fig. 9 GL-model, $q S$ wave: phase velocity $v$ and attenuation factor $k^{(\Im)} v s$ the propagation angle $\theta$ and the angular frequency $\omega$. Relaxation times $t_{0}=1 \times 10^{-12} \mathrm{~s}$ and $t_{1}=40 t_{0}$.

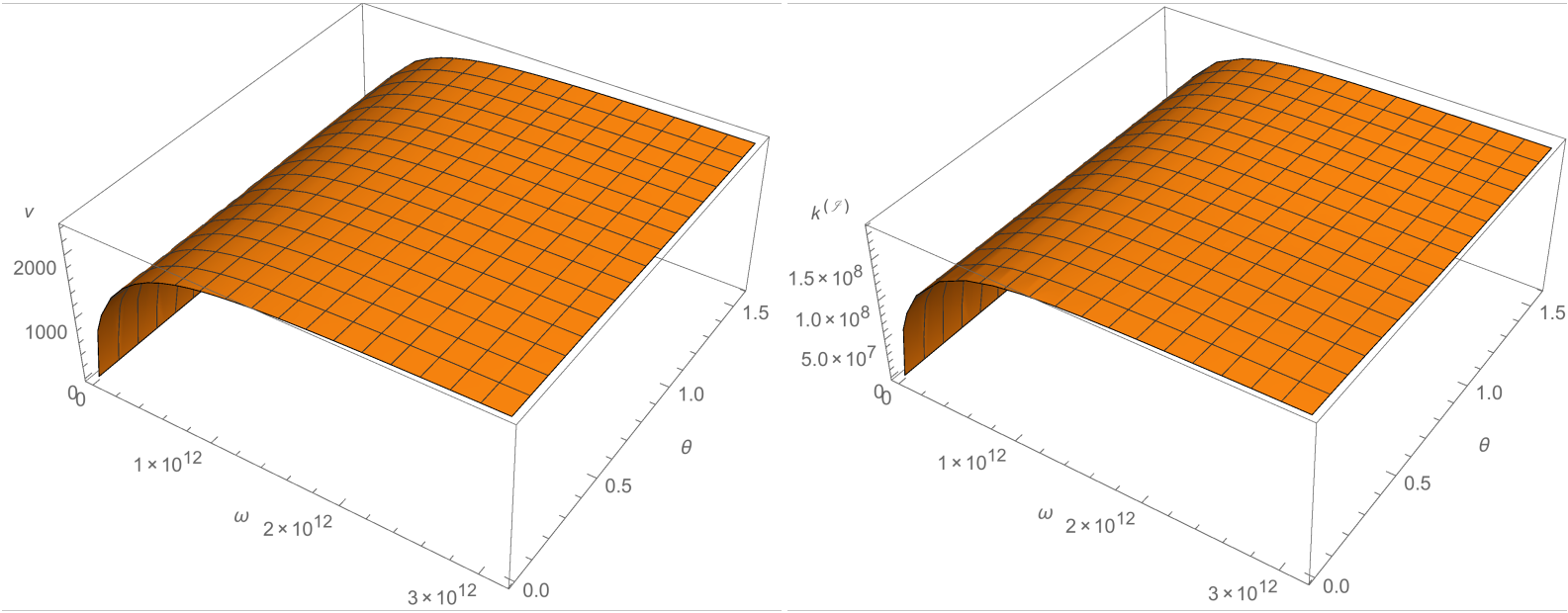

Fig. 10 GL-model, thermal wave: phase velocity $v$ and attenuation factor $k^{(\Im)} v s$ the propagation angle $\theta$ and the angular frequency $\omega$. Relaxation times $t_{0}=1 \times 10^{-12} \mathrm{~s}$ and $t_{1}=40 t_{0}$.

\subsection{Free boundary solution: reflection coefficients}

Although the dissipative feature of Eqs. (6) and considering that the medium anisotropy [45,47] imply that the mechanical components of $q P$ and $q S$ waves are not exactly longitudinal or transverse, we consider with good approximation merely longitudinal and transverse mechanical components, given the negligible phase shift (Fig. 13). Furthermore, we assume also the attenuation negligible and the propagation velocity independent of the angular frequency $\omega$ as previously remarked. In the following analysis, it should be noted that the quasi-thermal solution has been neglected, the mechanical components being too small if compared to those of $q P$ and $q S$ solutions.

Referring to Fig. 1 and labelling with $(0)$ the incident wave, let us assume a $q P$ wave travelling through the medium in the half space $z \leqslant 0$, with a propagation direction forming an angle $\theta_{0}$ with the $z$-axis and incident on the free interface. The incident wave is expressed through Eqs. (12) for a $q P$ wave in the $x z$-plane 

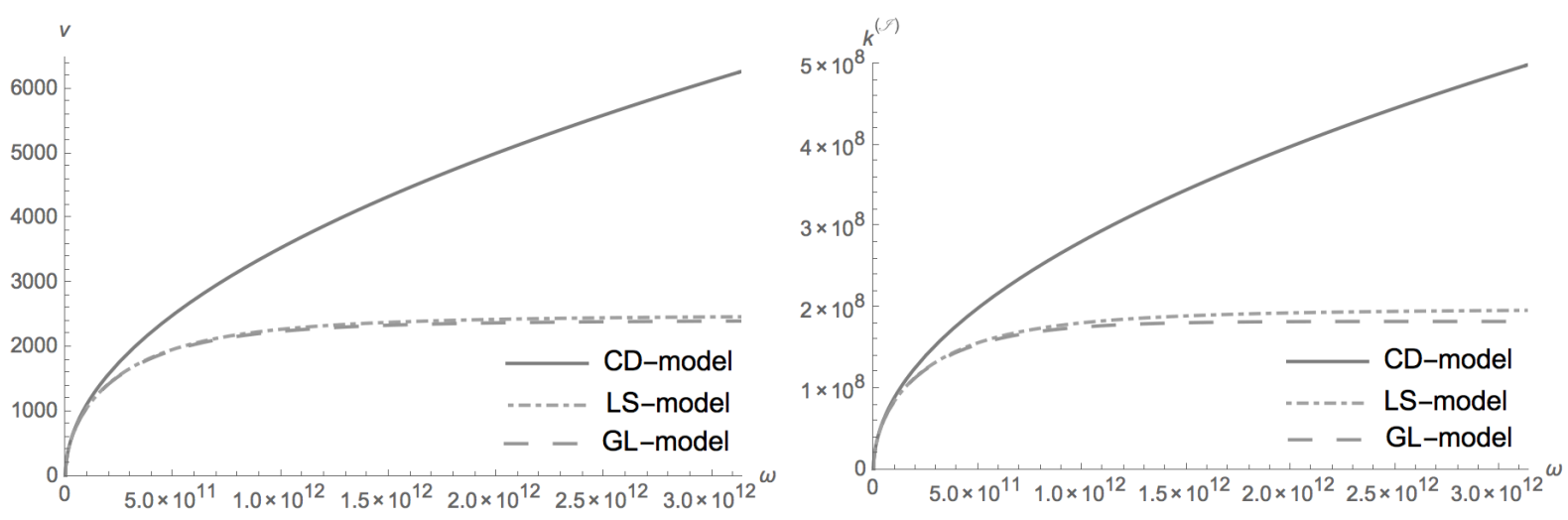

Fig. 11 Comparison among models: phase velocities $v$ and attenuation factors $k^{(\Im)}$ of the thermal wave. Relaxation times $t_{0}=1 \times 10^{-12}$ s and $t_{1}=40 t_{0}$.
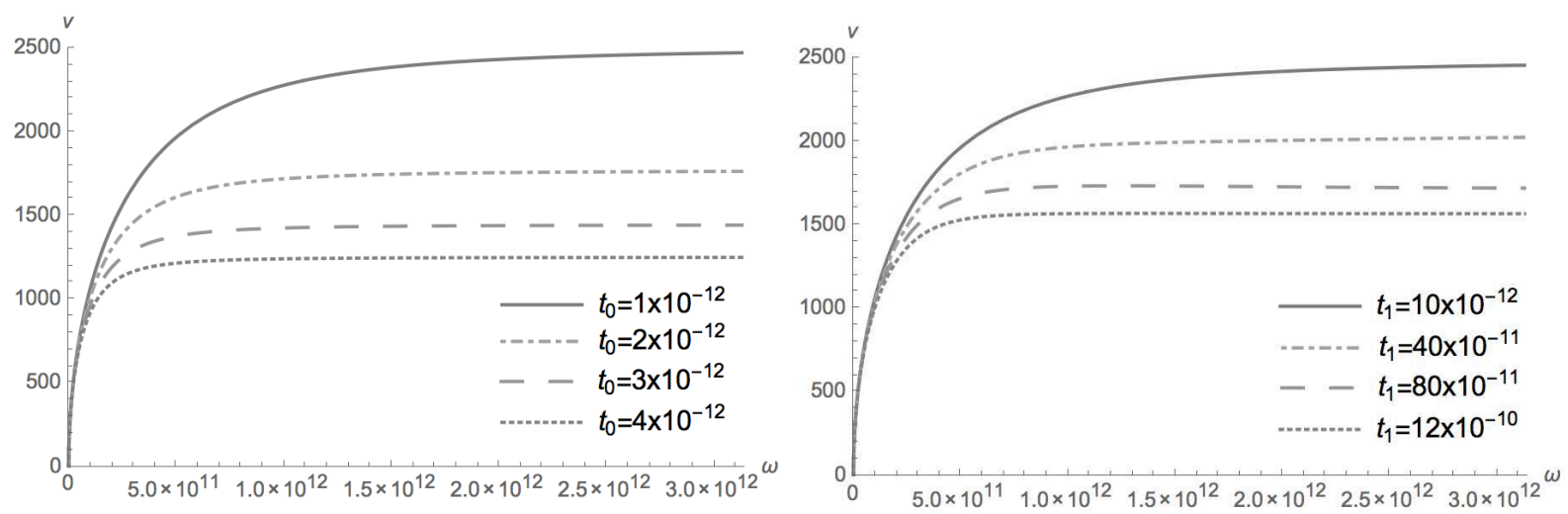

Fig. 12 Parametric analysis of phase velocities $v$ : LS-model (left) for various values of relaxation time $t_{0}$ and GL-model (right) for $t_{0}=1 \times 10^{-12} \mathrm{~s}$ and various values of $t_{1}$.
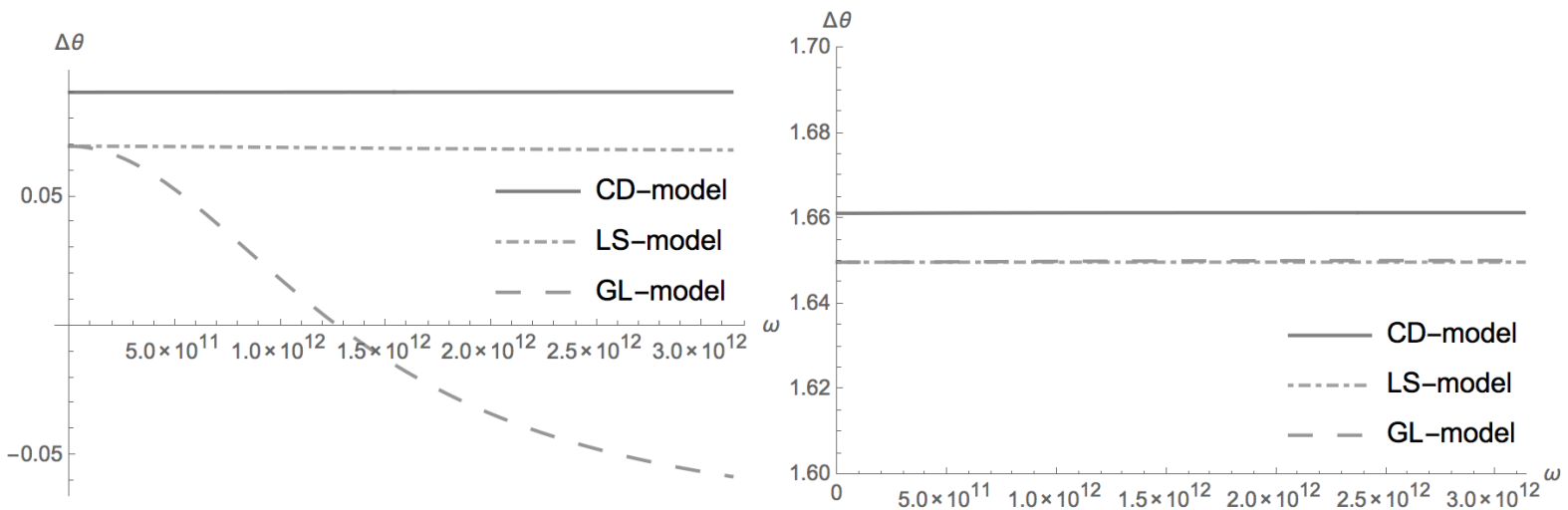

Fig. 13 Comparison among models for $\theta=\pi / 6$ : angle $\Delta \theta$ between displacement of the medium and direction of travel of elastic component for $q P$ waves (left) and $q S$ wave (right). Relaxation times $t_{0}=1 \times 10^{-12} \mathrm{~s}$ and $t_{1}=40 t_{0}$. 
with a displacement vector exactly parallel to $\mathbf{n}^{(0)}$, a unit propagation vector $\mathbf{n}^{(0)}=\left(\sin \theta_{0}, 0, \cos \theta_{0}\right)$ and for a null attenuation factor $k_{0}^{(\Im)}$ :

$$
\left\{\begin{array}{l}
\mathbf{u}^{(0)}=\left(u_{1}^{(0)}, u_{3}^{(0)}\right)=A_{0}\left(\sin \theta_{0}, \cos \theta_{0}\right) \exp \left[i k_{0}^{(\Re)}\left(x \sin \theta_{0}+z \cos \theta_{0}-v_{P 0} t\right)\right] \\
\varphi^{(0)}=B_{0} \exp \left[i k_{0}^{(\Re)}\left(x \sin \theta_{0}+z \cos \theta_{0}-v_{P 0} t\right)\right] \\
\vartheta^{(0)}=C_{0} \exp \left[i k_{0}^{(\Re)}\left(x \sin \theta_{0}+z \cos \theta_{0}-v_{P 0} t\right)\right]
\end{array}\right.
$$

where $A_{0}, B_{0}$ and $C_{0}$ are the amplitudes of the components and the propagation velocity is written as $v_{P 0}=\omega / k_{0}^{(\Re)}$.

Indicating with the label $(1)$ the reflected $q P$ wave also in this case with the displacement purely longitudinal, the unit propagation vector is $\mathbf{n}^{(1)}=\left(\sin \theta_{1}, 0,-\cos \theta_{1}\right)$ and, with analogous notation, one may write:

$$
\left\{\begin{array}{l}
\mathbf{u}^{(1)}=\left(u_{1}^{(1)}, u_{3}^{(1)}\right)=A_{1}\left(\sin \theta_{1},-\cos \theta_{1}\right) \exp \left[i k_{1}^{(\Re)}\left(x \sin \theta_{1}-z \cos \theta_{1}-v_{P 1} t\right)\right] \\
\varphi^{(1)}=B_{1} \exp \left[i k_{1}^{(\Re)}\left(x \sin \theta_{1}-z \cos \theta_{1}-v_{P 1} t\right)\right] \\
\vartheta^{(1)}=C_{1} \exp \left[i k_{1}^{(\Re)}\left(x \sin \theta_{1}-z \cos \theta_{1}-v_{P 1} t\right)\right]
\end{array}\right.
$$

where $v_{P 1}=\omega / k_{1}^{(\Re)}$.

In the same way using the label (2) for the reflected $q S$ wave, the unit propagation vector is $\mathbf{n}^{(2)}=$ $\left(\sin \theta_{2}, 0,-\cos \theta_{2}\right)$ and it is perpendicular to the unit displacement vector $\left(\cos \theta_{2}, 0, \sin \theta_{2}\right)$; so, we write:

$$
\left\{\begin{array}{l}
\mathbf{u}^{(2)}=\left(u_{1}^{(2)}, u_{3}^{(2)}\right)=A_{2}\left(\cos \theta_{2}, \sin \theta_{2}\right) \exp \left[i k_{2}^{(\Re)}\left(x \sin \theta_{2}-z \cos \theta_{2}-v_{S 2} t\right)\right] \\
\varphi^{(2)}=B_{2} \exp \left[i k_{2}^{(\Re)}\left(x \sin \theta_{2}-z \cos \theta_{2}-v_{S 2} t\right)\right] \\
\vartheta^{(2)}=C_{2} \exp \left[i k_{2}^{(\Re)}\left(x \sin \theta_{2}-z \cos \theta_{2}-v_{S 2} t\right)\right]
\end{array}\right.
$$

where $v_{S 2}=\omega / k_{2}^{(\Re)}$.

Let us substitute the waves represented in Eqs. (17), (18) and (19) in the boundary conditions (7), (8) and (9) at the interface $z=0$, defining the exponent of waves as

$$
i \bar{\eta}_{n}=i k_{n}^{(\Re)}\left(x \sin \theta_{n}-v_{H n} t\right),
$$

where the index $n$ labels the various types of waves and $H$ stands for $P$ or $S$. Considering that all Eqs. (7), (8) and (9) must be satisfied for every value of $x$ and $t$, necessarily we have:

$$
\bar{\eta}_{0}=\bar{\eta}_{1}=\bar{\eta}_{2}
$$

that implies

$$
\left\{\begin{array}{l}
k_{0}^{(\Re)} \sin \theta_{0}=k_{1}^{(\Re)} \sin \theta_{1}=k_{2}^{(\Re)} \sin \theta_{2} \\
k_{0}^{(\Re)} v_{P 0}=k_{1}^{(\Re)} v_{P 1}=k_{2}^{(\Re)} v_{S 2}=\omega
\end{array}\right.
$$

From them and the transverse isotropy properties, we obtain for the incident and reflected $q P$ waves,

$$
k_{0}^{(\Re)}=k_{1}^{(\Re)}, \quad \theta_{0}=\theta_{1}, \quad v_{P 0}=v_{P 1},
$$

while between the reflected waves we set,

$$
\frac{k_{2}^{(\Re)}}{k_{1}^{(\Re)}}=\frac{v_{P 1}}{v_{S 2}}=\tau_{1}, \quad \sin \theta_{2}=\frac{1}{\tau_{1}} \sin \theta_{0}
$$


Substituting Eqs. (17), (18) and (19) for incident and reflected waves into the two-dimensional Eq. (6) 1 and simplifying the exponentials, we get the following relations among the wave amplitudes

$$
\kappa_{n} A_{n}+M_{n} B_{n}+\mu_{n} C_{n}=0 \quad \text { with } n=0,1,2
$$

where the parameters $\kappa_{n}, M_{n}$ and $\mu_{n}$ are defined by the relations (34), (35) and (36) in Appendix A. Eqs. (25) represent a system of homogeneous linear equations that defines the relations among the amplitudes of the elastic, electric and thermal components. From Eqs. (34), (35) and (36), one may obtain the following relations:

$$
\kappa_{0}=-\kappa_{1}, \quad M_{0}=M_{1}, \quad \mu_{0}=-\mu_{1}
$$

Substituting Eqs. (17), (18) and (19) in the Gauss Eq. (6) 2 and simplifying the exponentials, one obtains:

$$
L_{n} A_{n}+G_{n} B_{n}+S_{n} C_{n}=0 \quad \text { with } n=0,1,2
$$

where the parameters $L_{n}, G_{n}$ and $S_{n}$ are defined by the relations (37), (38) and (39) in the Appendix A and obey to the following relations:

$$
L_{0}=-L_{1}, \quad G_{0}=G_{1}, \quad S_{0}=-S_{1}
$$

In the same way, by the substitution of Eqs. (17), (18) and (19) into the heat conduction Eq. (6) ${ }_{3}$, it yields:

$$
E_{n} A_{n}+D_{n} B_{n}+F_{n} C_{n}=0 \quad \text { with } n=0,1,2
$$

where the parameters $E_{n}, D_{n}$ and $F_{n}$ are defined by the relations (40), (41) and (42) in Appendix A. From these equations, one obtains the following relations:

$$
E_{0}=-E_{1}, \quad D_{0}=D_{1}, \quad F_{0}=-F_{1}
$$

From the above considerations, we have a set of nine linear, algebraic, non-independent, homogeneous equations, connected by Eqs. (25) Eqs. (27) Eqs. (29), in the six unknowns $A_{n}, B_{n}$ and $C_{n}$ (with $n=1,2$ ). Due to Eq. (14), one obtains non trivial relations for the reflection coefficients. We have:

$$
X_{1}=\frac{A_{1}}{A_{0}}=\frac{T_{1}+T_{2}}{T_{1}-T_{2}}, \quad X_{2}=\frac{A_{2}}{A_{0}}=\frac{2}{T_{2}-T_{1}},
$$

for the elastic components,

$$
Z_{1}=\frac{B_{1}}{B_{0}}=-\frac{A_{1}}{A_{0}}, \quad Z_{2}=\frac{B_{2}}{B_{0}}=\frac{A_{1}}{A_{0}}-1,
$$

for the electric components and

$$
W_{1}=\frac{C_{1}}{C_{0}}=\frac{A_{1}}{A_{0}}, \quad W_{2}=\frac{C_{2}}{C_{0}}=-\frac{A_{1}}{A_{0}}-1,
$$

for the thermal components, where the auxiliary coefficients $T_{1}$ and $T_{2}$ are defined by the relations (43) and (44) in Appendix A. Hence, when a $q P$-wave impinges upon a free interface of a pyroelectric medium with an angle of incidence equal to $\theta_{0}$, it originates two reflected waves: a $q P$-wave with a reflection angle $\theta_{1}=\theta_{0}$ and a $q S$-wave with a reflection angle $\theta_{2}=\arcsin \left[\left(1 / \tau_{1}\right) \sin \theta_{0}\right]$ (Eqs. (23) and (24)). Each wave has mechanical, electric and thermal components and the relative reflection coefficients are characterised by Eqs. (31), (32) and (33).

As a special case, if we neglect the electrical constants, i.e. we set $e_{15}=e_{31}=e_{33}=d_{3}=0, p_{11}=p_{33}$, the reflection coefficients provided by Eqs. (31), (32) and (33) reduce to those in [56], where wave reflection is studied in a transversely isotropic, thermoelastic medium employing nonclassical theory of thermoelasticity. 


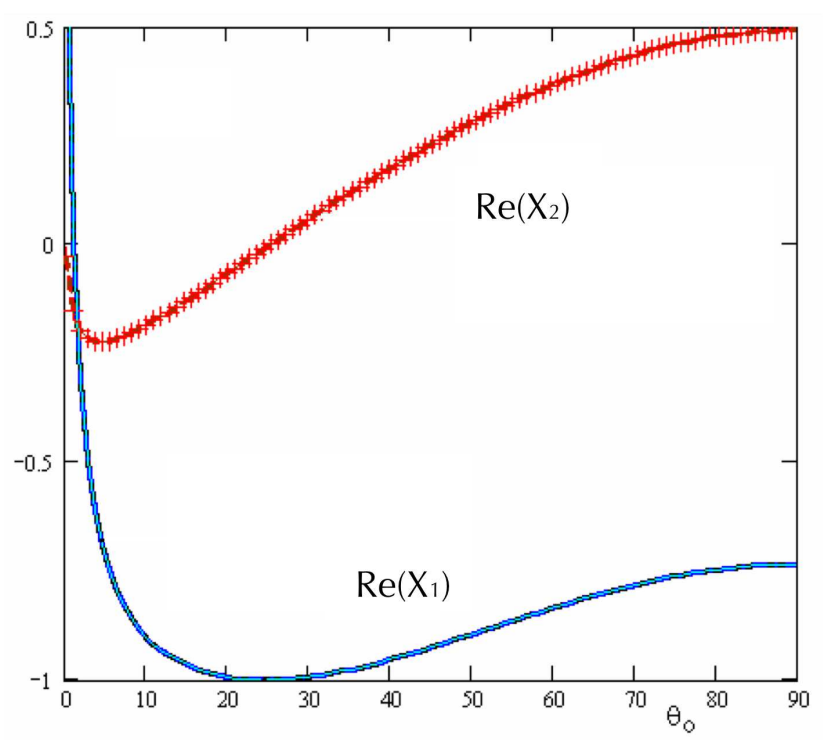

Fig. 14 CD-model, LS-model, GL-model: coinciding real parts of the reflection coefficients $X_{1}$ and $X_{2}$ versus $\theta_{0}$ with $t_{0}=t_{1}=1 \times 10^{-12} \mathrm{~s}$.

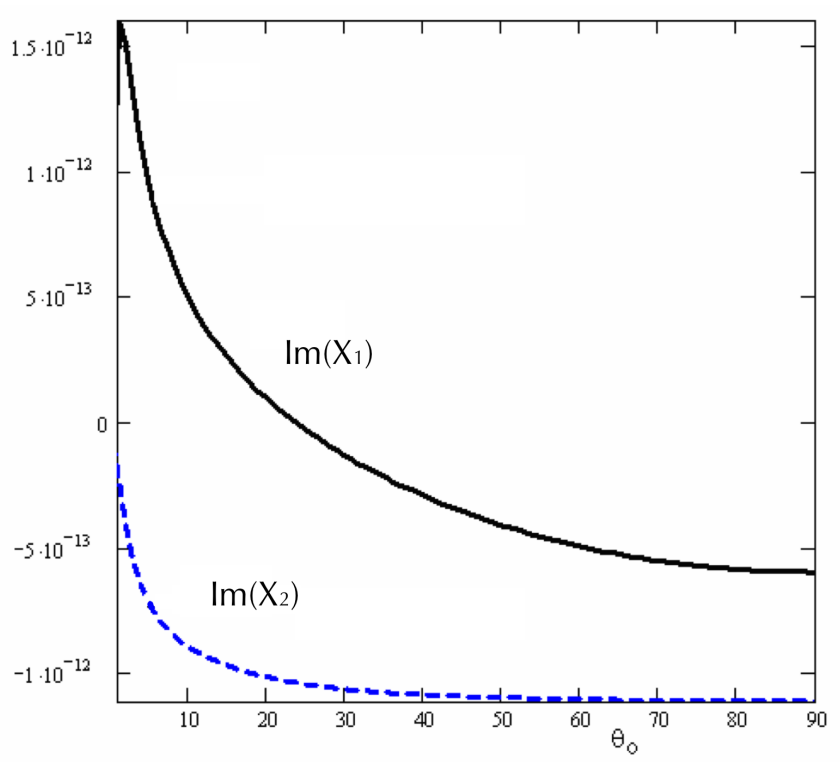

Fig. 15 CD-model: imaginary parts of reflection coefficients $X_{1}$ and $X_{2}$ versus $\theta_{0}$.

\section{Discussion and Conclusions}

Using the physical constants in Table 1, the reflection coefficients are computed as functions of the angle of incidence $\theta_{0}$. The results obtained for the real and imaginary parts of the mechanical reflection coefficients $X_{1}$ and $X_{2}$ (Eq. 31) are reported in Figs. 14, 15, 16 and 17, for a few values of the thermal relaxation times $t_{0}$ and $t_{1}$. With the hypothesis previously made, the graphs are valid for any angular frequency $\omega$. 

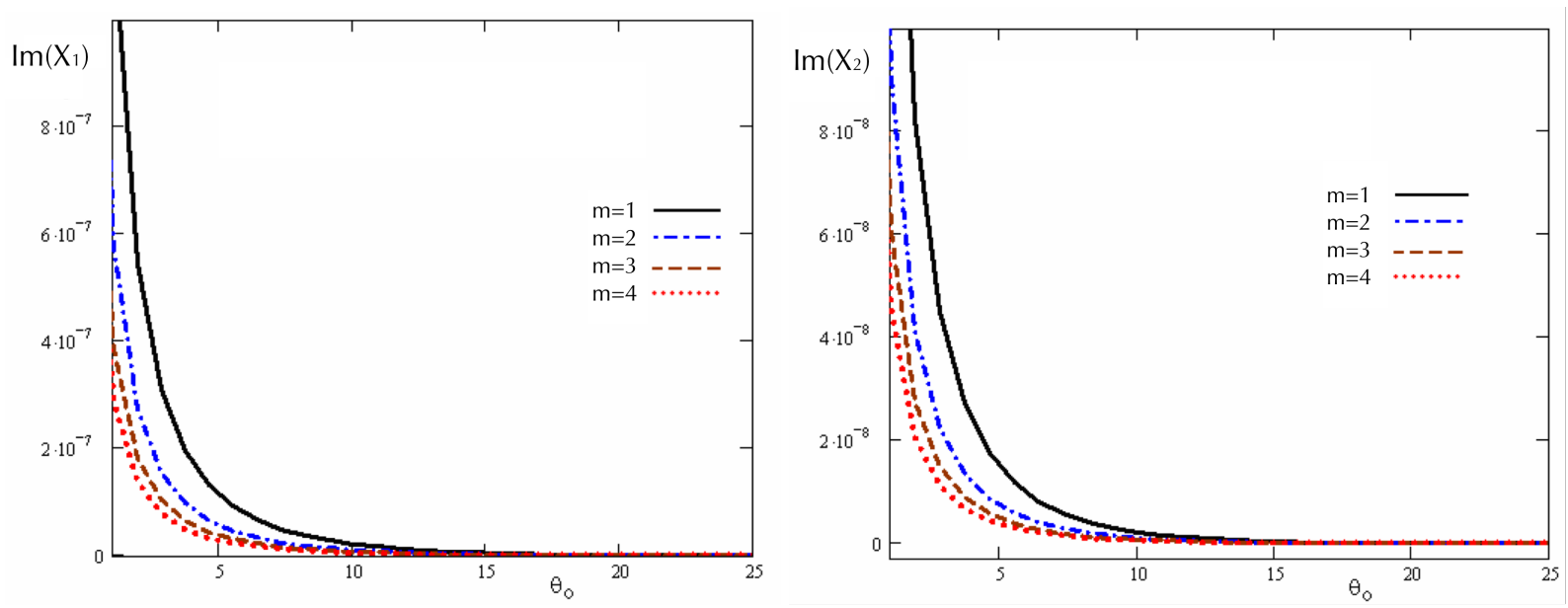

Fig. 16 LS-model: imaginary parts of the reflection coefficients $X_{1}$ and $X_{2}$ versus $\theta_{0}$ for different values of the relaxation time $t_{0}=m \times 10^{-12} \mathrm{~s}$.
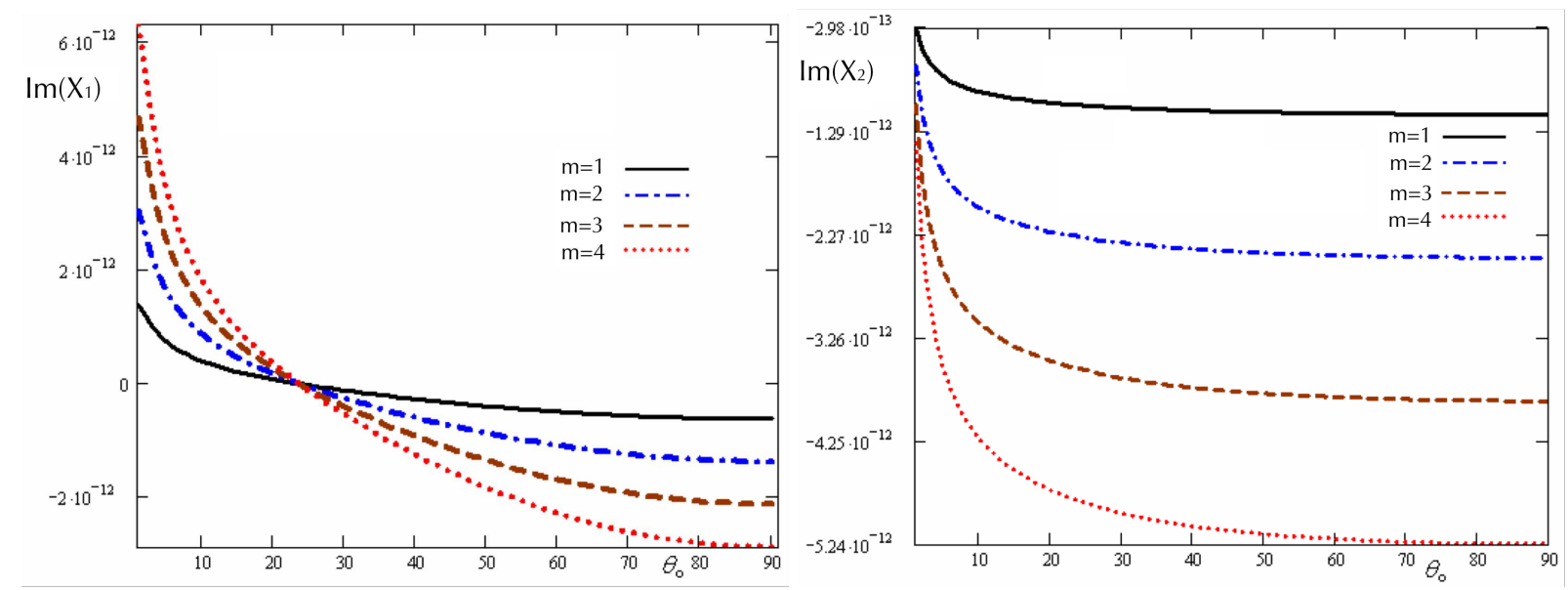

Fig. 17 GL-model: imaginary parts of the reflection coefficients $X_{1}$ and $X_{2}$ versus $\theta_{0}$ for different values of the relaxation times $t_{0}=1 \times 10^{-12} \mathrm{~s}$ and $t_{1}=m t_{0}$.

From an overall observation, we note in Figs. 15, 16 and 17 that the imaginary parts $\Im\left(X_{i}\right)$ and $\Im\left(X_{2}\right)$ decrease monotonously with increasing $\theta_{0}$; each investigated model shows a stationary trend for $\theta \rightarrow 90^{\circ}$, tending to a value which in independent from $t_{0}$ for LS-model and, conversely, strongly dependent for GL-model. $\Re\left(X_{1}\right)$ and $\Re\left(X_{2}\right)$, reported for all three models in the same Fig. 14 because coinciding, show a minimum at about $\theta_{0} \approx 25^{\circ}$ for $\Re\left(X_{1}\right)$ and about $\theta_{0} \approx 5^{\circ}$ for $\Re\left(X_{2}\right)$.

An interesting behaviour is observed for all the models considered: at about $\theta_{0} \approx 25^{\circ}, \Re\left(X_{1}\right)=-1$ and $\Re\left(X_{2}\right)=0$, while $\Im\left(X_{i}\right) \approx 0$, i.e. at this value of the incident angle the reflected $q S$-wave is absent and therefore only the $q P$-wave is observed.

Finally it is worth emphasising that previous studies, e.g. Achenbach [6] and Abd-alla et al. [3], can be considered as special cases of the problem formulated in this work.

In this paper, we have studied the reflection of linear waves at the boundary separating a vacuum and a half-space occupied by an elastic, transversely isotropic, thermo-piezoelectric material under the influence 
of thermal relaxation times. The classical Fourier description of heat conduction has been compared with non-classical theories, namely the Lord-Shulman and the Green-Lindsay models, characterised respectively by one and two relaxation times. The proposed two-dimensional model indicates that the reflection of a $q P$ wave, at the boundary between the vacuum and the medium, generates three different wave solutions, referred to as a $q P$ wave, a $q S$ wave and a $q T$ wave. The latter can be neglected since it has mechanical components little significant. For the non-classical models considered, a finite propagation velocity has been found, coherently to the hyperbolic nature of the modified heat equation.

The analytical results obtained were applied to compute the reflection coefficients for the specific pyroelectric material cadmium selenide $(C d S e)$. The results determined show a dependence on the relaxation times for the Green-Lindsay model and illustrate a particular circumstance for which the $q S$-wave is absent. We reckon that this study will be useful in signal processing, sound systems and wireless communication, in addition to the design and construction of gyroscopes, temperature sensors, pyroelectric surface acoustic wave (SAW) devices and defence systems.

\section{A Appendix}

Herein, the auxiliary parameters used in computation are listed:

$$
\begin{aligned}
& \kappa_{0}=\sin \theta_{0}\left(\rho v_{P 0}^{2}-c_{11} \sin ^{2} \theta_{0}-\left(c_{13}+2 c_{44}\right) \cos ^{2} \theta_{0}\right) \\
& M_{0}=-\left(e_{15}+e_{13}\right) \sin \theta_{0} \cos \theta_{0} \\
& \mu_{0}=-\gamma_{1}\left(\frac{i}{k_{0}^{(\Re)}}\right) \sin \theta_{0}\left(1-i k_{0}^{(\Re)} v_{P 0} t_{1}\right) \\
& \kappa_{1}=\sin \theta_{1}\left(c_{11} \sin ^{2} \theta_{1}+\left(c_{13}+2 c_{44}\right) \cos ^{2} \theta_{1}-\rho v_{P 1}^{2}\right) \\
& M_{1}=-\left(e_{13}-e_{15}\right) \sin \theta_{1} \cos \theta_{1} \\
& \mu_{1}=\gamma_{1} \sin \theta_{1}\left(\frac{i}{k_{1}^{(\Re)}}\right)\left(1-i k_{1}^{(\Re)} v_{P 1} t_{1}\right) \\
& \kappa_{2}=\cos \theta_{2}\left(c_{11} \sin ^{2} \theta_{2}-\left(c_{13}+c_{44}\right) \sin ^{2} \theta_{2}+c_{44} \cos ^{2} \theta_{2}-\rho v_{S 2}^{2}\right) \\
& M_{2}=-\left(e_{13}+e_{15}\right) \sin \theta_{2} \cos \theta_{2} \\
& \mu_{2}=\gamma_{1}\left(\frac{i}{k_{2}^{(\Re)}}\right) \sin \theta_{2}\left(1-i k_{2}^{(\Re)} v_{S 2} t_{1}\right) \\
& L_{0}=-\left(\left(e_{13}+2 e_{15}\right) \sin ^{2} \theta_{0} \cos \theta_{0}+e_{33} \cos ^{3} \theta_{0}\right) \\
& G_{0}=\left(p_{11} \sin ^{2} \theta_{0}+p_{33} \cos ^{2} \theta_{0}\right) \\
& S_{0}=d_{3}\left(\frac{i}{k_{0}^{(\Re)}}\right) \cos \theta_{0}\left(1-i k_{0}^{(\Re)} t_{1} v_{P 0}\right) \\
& L_{1}=\left(\left(e_{13}+2 e_{15}\right) \sin ^{2} \theta_{1} \cos \theta_{1}+e_{33} \cos ^{3} \theta_{1}\right) \\
& G_{1}=\left(p_{11} \sin ^{2} \theta_{1}+p_{33} \cos ^{2} \theta_{1}\right) \\
& S_{1}=-d_{3}\left(\frac{i}{k_{1}^{(\Re)}}\right) \cos \theta_{1}\left(1-i k_{1}^{(\Re)} t_{1} v_{P 1}\right) \\
& L_{2}=\left(e_{15}\left(\sin \theta_{2} \cos ^{2} \theta_{2}-\sin ^{3} \theta_{2}\right)+\left(e_{13}-e_{33}\right) \sin \theta_{2} \cos ^{2} \theta_{2}\right) \\
& G_{2}=\left(p_{11} \sin ^{2} \theta_{2}+p_{33} \cos ^{2} \theta_{2}\right) \\
& S_{2}=-d_{3}\left(\frac{i}{k_{2}^{(\Re)}}\right) \cos \theta_{2}\left(1-i k_{2}^{(\Re)} t_{1} v_{S 2}\right) \\
& E_{0}=T_{0}\left(\left(1-i t_{0} \delta k_{0}^{(\Re)} v_{P 0}\right)\left(\gamma_{1} \sin ^{2} \theta_{0}+\gamma_{3} \cos ^{2} \theta_{0}\right)\right) \\
& D_{0}=-T_{0}\left(d_{3} \cos \theta_{0}\left(1-i t_{0} \delta k_{0}^{(\Re)} v_{P 0}\right)\right) \\
& F_{0}=\left(\frac{K_{11}}{v_{P 0}} \sin ^{2} \theta_{0}+\frac{K_{33}}{v_{P 0}} \cos ^{2} \theta_{0}-\rho C_{\varepsilon}\left(1-t_{0} i k_{0}^{(\Re)} v_{P 0}\right)\right) \\
& E_{1}=-T_{0}\left(\left(1-t_{0} \delta i k_{1}^{(\Re)} v_{P 1}\right)\left(\gamma_{1} \sin ^{2} \theta_{1}+\gamma_{3} \cos ^{2} \theta_{1}\right)\right) \\
& D_{1}=-T_{0}\left(d_{3} \cos \theta_{1}\left(1-t_{0} \delta i k_{1}^{(\Re)} v_{P 1}\right)\right) \\
& F_{1}=-\left(\frac{K_{11}}{v_{P 1}} \sin ^{2} \theta_{1}+\frac{K_{33}}{v_{P 1}} \cos ^{2} \theta_{1}-\rho C_{\varepsilon}\left(1-t_{0} i k_{1}^{(\Re)} v_{P 1}\right)\right) \\
& E_{2}=-T_{0}\left(\left(1-t_{0} \delta i k_{2}^{(\Re)} v_{S 2}\right)\left(\left(\gamma_{1}-\gamma_{3}\right) \sin \theta_{2} \cos \theta_{2}\right)\right. \\
& D_{2}=-T_{0}\left(d_{3} \cos \theta_{2}\left(1-t_{0} \delta i k_{2}^{(\Re)} v_{S 2}\right)\right) \\
& F_{2}=-\left(\frac{K_{11}}{v_{S 2}} \sin ^{2} \theta_{2}+\frac{K_{33}}{v_{S 2}} \cos ^{2} \theta_{2}-\rho C_{\varepsilon}\left(1-t_{0} i k_{2}^{(\Re)} v_{S 2}\right)\right)
\end{aligned}
$$




$$
\begin{gathered}
T_{1}=\frac{\tau_{1}\left(D_{0} \mu_{0}-F_{0} M_{0}\right)\left[\left(D_{2} \mu_{2}-F_{2} M_{2}\right)\left(c_{13}-c_{33}\right) \sin \theta_{2} \cos \theta_{2}-\left(E_{2} \mu_{2}-F_{2} \kappa_{2}\right) e_{33} \cos \theta_{2}\right]}{\left(D_{2} \mu_{2}-F_{2} M_{2}\right)\left[\left(D_{0} \mu_{0}-F_{0} M_{0}\right)\left(c_{13} \sin ^{2} \theta_{0}+c_{33} \cos ^{2} \theta_{0}\right)+\left(E_{0} \mu_{0}-F_{0} \kappa_{0}\right) e_{33} \cos \theta_{0}\right]} \\
T_{2}=\frac{\tau_{1}\left(D_{0} \mu_{0}-F_{0} M_{0}\right)\left[\left(D_{2} \mu_{2}-F_{2} M_{2}\right) c_{44} \cos 2 \theta_{2}-\left(E_{2} \mu_{2}-F_{2} \kappa_{2}\right) e_{15} \sin \theta_{2}\right]}{\left(D_{2} \mu_{2}-F_{2} M_{2}\right)\left[\left(D_{0} \mu_{0}-F_{0} M_{0}\right) c_{44} \sin 2 \theta_{0}+\left(E_{0} \mu_{0}-F_{0} \kappa_{0}\right) e_{15} \cos \theta_{0}\right]}
\end{gathered}
$$

\section{References}

1. Abd-alla, A.N. and Yahia, A. and Abo-dahab, S.: On the reflection of the generalized magneto-thermo-viscoelastic plane waves. Chaos, Solitons and Fractals 16, 211-231 (2003). 2

2. Abd-Alla, A. N. and Abo-dahab, S. M.: The influence of the viscosity on reflection and refraction of plane shear elastic waves in two magnetized semi-infinite media is investigated. Meccanica 43(4), 437-448 (2008). 2

3. Abd-alla, A.N. and Al-sheikh, F.A. and Al-Hossain, A.Y.: The reflection phenomena of quasi-vertical transverse waves in piezoelectric medium under initial stresses. Meccanica 47(3), 731-744 (2012). 3, 17

4. Abd-alla, A.N. and Al-Hossain, A.Y. and Elhaes, H. and Ibrahim, M.: Reflection and Refraction of Waves in NanoSmart Materials: Anisotropic Thermo-piezoelectric Materials. Journal of Computational and Theoretical Nanoscience 11(3), 715-726 (2014). 3

5. Abd-alla, A.N. and Hamdan, A. M. and Giorgio, I. and Del Vescovo, D.: The Mathematical Model of Reflection and Refraction of Longitudinal Waves in Thermo-piezoelectric Materials. Archive of Applied Mechanics, (2014). DOI:10.1007/s00419-014-0852-z. 3

6. Achenbach, J.D.: Wave Propagation in Elastic Solids. North-Holland Series in Applied Mathematics and Mechanics 16, New York (1973). 2, 17

7. Andreaus, U., Chiaia, B. and Placidi L.: Soft-impact dynamics of deformable bodies. Continuum Mechanics and Thermodynamics 25, 375-398 (2013), DOI: 10.1007/s00161-012-0266-5 3

8. Andreaus, U., dell'Isola, F., Porfiri, M.: Piezoelectric passive distributed controllers for beam flexural vibrations. JVC/Journal of Vibration and Control 10(5), 625-659 (2004). 2

9. Auld, B.: Acoustic Field and Waves in Solids. R. E. Krieger Pub. Com., Malabar, Florida, U.S.A. (1990). 2,7

10. Berezovski A. and Maugin G.A.: Thermoelastic wave and front propagation. Journal of Thermal Stresses 25(8), 719-743 (2002). 3

11. Boehler, J. P.: Representations for isotropic and anisotropic non-polynomial tensor functions. Applications of tensor functions in solid mechanics, edited by J. P. Boehler, CISM Courses and Lectures 292, Springer, Wien, 31-53 (1987). 7

12. Carcaterra, A. and Roveri, N. and Pepe, G.: Fractional dissipation generated by hidden wave-fields. Mathematics and Mechanics of Solids, (2014). DOI: 10.1177/1081286513518941. 3

13. Carcaterra, A. and Roveri, N.: Energy distribution in impulsively excited structures, Shock and Vibration 19(5), 11431163 (2012). 3

14. Cazzani, A. and Lovadina, C.: On some mixed finite element methods for plane membrane problems. Computational Mechanics 20(6), 560-572 (1997). 11

15. Cuomo, M. and Greco, L.: Isogeometric analysis of space rods: Considerations on stress locking. ECCOMAS $2012-$ European Congress on Computational Methods in Applied Sciences and Engineering, e-Book Full Papers, 5094-5112 (2012). 11

16. dell'Isola, F. and Madeo, A. and Seppecher, P.: Boundary conditions at fluid-permeable interfaces in porous media: A variational approach. International Journal of Solids and Structures 46(17):3150-3164 (2009). 3

17. dell'Isola, F. and Madeo, A. and Placidi, L.: Linear plane wave propagation and normal transmission and reflection at discontinuity surfaces in second gradient 3D Continua. ZAMM - Journal of Applied Mathematics and Mechanics 92(1), $52-71(2012) .3$

18. dell'Isola, F., Sciarra, G. and Vidoli, S.: Generalized Hooke's law for isotropic second gradient materials. Proceedings of the Royal Society A: Mathematical, Physical and Engineering Sciences 465(2107), 2177-2196 (2009). 3

19. dell'Isola, F. and Vidoli, S.: Continuum modelling of piezoelectromechanical truss beams: An application to vibration damping. Archive of Applied Mechanics 68(1), 1-19 (1998). 2

20. Del Vescovo, D., and Giorgio, I.: Dynamic problems for metamaterials: Review of existing models and ideas for further research. International Journal of Engineering Science, (2014). http://dx.doi.org/10.1016/j.ijengsci.2014.02.022. 6

21. Eremeyev, V. A.: Acceleration waves in micropolar elastic media, Doklady Physics 50(4), 204-206 (2005). 3

22. Greco, L. and Cuomo, M.: B-Spline interpolation of Kirchhoff-Love space rods. Computer Methods in Applied Mechanics and Engineering 256, 251-269 (2013). 11

23. Greco, L. and Cuomo, M.: An implicit G1 multi patch B-spline interpolation for Kirchhoff-Love space rod. Computer Methods in Applied Mechanics and Engineering 269, 173-197 (2014). 11

24. Green A. and Lindsay K. A.: Thermoelasticity. J. Elasticity 2, 1-7 (1972). 2, 5

25. Guo, S. H.: The thermo-electromagnetic waves in piezoelectric solids. Acta Mech. 219, 231-240 (2011). 2

26. Huang, H.T. and Zhou, L.M.: Micromechanics approach to the magnetoelectric properties of laminate and fibrous piezoelectric/magnetostrictive composites. J. Phys. D: Appl. Phys. 37, 3361-3366 (2004). 2 
27. Lubarda V.A. and Chen M.C.: On the elastic moduli and compliances of transversely isotropic and orthotropic materials. J. Mech. Materials and Structures 3(1), 153-171 (2008). 5, 7

28. Luongo, A., Paolone, A. and Piccardo, G.: Postcritical behavior of cables undergoing two simultaneous galloping modes. Meccanica 33(3), 229-242 (1998). 3

29. Luongo, A. and Piccardo, G.: Linear instability mechanisms for coupled translational galloping. J. Sound Vib. 288(4-5), 1027-1047 (2005). 3

30. Keith, C.M. and Crampin, S.: Seismic body waves in anisotropic media: reflection and refraction at a plane interface, Geophysical Journal of the Royal Astronomical Society 49(1), 181-208 (1977). 3

31. Kuang, Z. B. and Yuan, X. G.: Reflection and transmission of waves in pyroelectric and piezoelectric materials, J. of Sound and Vibration 330(6), 1111-1120 (2011). 3

32. Rupender, K. R.: Propagation of waves in an electro-microstretch generalized thermoelastic semi-space. Acta Mech Sinica 25(5), 619-628 (2009). 3

33. Kumar, R. and Kumar, R.: Wave propagation at the boundary surface of elastic and initially stressed visco-thermoelastic diffusion with voids media. Meccanica 48(9), 2173-2188 (2013). 3

34. Madeo, A., Neff, P., Ghiba, I.D., Placidi, L. and Rosi, G.: Wave propagation in relaxed micromorphic continua: modeling metamaterials with frequency band-gaps. Continuum Mechanics and Thermodynamics, (2013). DOI:10.1007/s00161013-0329-2. 3

35. Maurini, C., Pouget, J. and dell'Isola, F.: On a model of layered piezoelectric beams including transverse stress effect. International Journal of Solids and Structures 41(16-17), 4473-4502 (2004). 2

36. Maurini, C., Pouget, J. and dell'Isola, F.: Extension of the Euler-Bernoulli model of piezoelectric laminates to include 3D effects via a mixed approach. Computers and Structures 84(22-23), 1438-1458 (2006). 2

37. Madeo, A., Djeran-Maigre, I., Rosi, G. and Silvani, C.: The effect of fluid streams in porous media on acoustic compression wave propagation, transmission, and reflection. Continuum Mechanics and Thermodynamics 6, 1-24 (2012).

38. Madeo, A. and dell'Isola, F. and Darve, F.: A continuum model for deformable, second gradient porous media partially saturated with compressible fluids. Journal of the Mechanics and Physics of Solids 61(11), 2196-2211 (2013). 3

39. Misra, A. and Marangos, O.: Parametric Studies of Wave Propagation Through Imperfect Interfaces Using Micromechanics Based Effective Stiffness. Review of Progress in Quantitative Nondestructive Evaluation 27B, 1074-1081 (2008).

40. Misra, A. and Marangos, O.: Effect of Contact Viscosity and Roughness on Interface Stiffness and Wave Propagation. Review of Progress in Quantitative Nondestructive Evaluation 28A, 105-112 (2009). 3

41. Nayfeh, A.H.: Wave Propagation in Layered Anisotropic Media. North-Holland, Amsterdam (1995). 2

42. Neff, P., Ghiba, I.D., Madeo, A., Placidi and L., Rosi, G.: A unifying perspective: The relaxed linear micromorphic continuum. Contin. Mech. and Thermodyn., (2013). DOI:10.1007/s00161-013-0322-9. 3

43. Othman, M. I. A. and Song, Y. Q.: Reflection of magneto-thermo-elastic waves from a rotating elastic half-space. Int. J. Eng. Sci. 46(5), 459-474 (2008). 2

44. Parton, V. and Kudryavtsev, B.: Electromagnetoelasticity: Piezoelectrics and Electrically Conductive Solids. Gordon \& Breach, NY, (1988). 2

45. Placidi L. and Hutter K.: An Anisotropic Flow Law for Incompressible Polycrystalline Materials. Zeitschrift Für Angewandte Mathematik und Physik - ZAMP 57, 160-181 (2006), DOI: 10.1007/s00033-005-0008-7 12

46. Placidi, L., dell'Isola, F., Ianiro, N. and Sciarra, G.: Variational formulation of pre-stressed solid-fluid mixture theory, with an application to wave phenomena. European Journal of Mechanics, A/Solids 27, 582-606 (2008). 3

47. Placidi L., Greve R., Seddik H. and Faria S.H.: Continuum-mechanical, Anisotropic Flow model, based on an anisotropic Flow Enhancement factor. Continuum Mechanics and Thermodynamics 22, 221-237 (2010), DOI: 10.1007/s00161-0090126-0 12

48. Placidi, L. and Rosi, G. and Giorgio, I. and Madeo, A.: Reflection and transmission of plane waves at surfaces carrying material properties and embedded in second-gradient materials. Mathematics and Mechanics of Solids 19(5), 555-578 (2014). 3

49. Placidi, L.: A variational approach for a nonlinear 1-dimensional second gradient continuum damage model. Continuum Mechanics and Thermodynamics 1-16, (2014). DOI: 10.1007/s00161-14-0338-9 3

50. Quiligotti, S. and Maugin, G.A. and dell'Isola, F.: Wave motions in unbounded poroelastic solids infused with compressible fluids. Zeitschrift Für Angewandte Mathematik und Physik - ZAMP 53(6), 1110-1113 (2002). 3

51. Reccia, E. and Cazzani, A. and Cecchi, A.: FEM-DEM Modeling for Out-of-plane Loaded Masonry Panels: A Limit Analysis Approach. Open Civil Engineering Journal 6(1), 231-238 (2012). 11

52. Rosi, G. and Madeo, A. and Guyader, J.L.: Switch between fast and slow Biot compression waves induced by second gradient microstructure at material discontinuity surfaces in porous media. International Journal of Solids and Structures 50(10), 1721-1746 (2013). 3

53. Rosi, G. and Giorgio, I. and Eremeyev, V. A.: Propagation of linear compression waves through plane interfacial layers and mass adsorption in second gradient fluids. ZAMM - Journal of Applied Mathematics and Mechanics 93(12), 914-927 (2013). DOI:10.1002/zamm.201200285. 3

54. Royer, D. and Dieulesaint, E.: Elastic Waves in Solids I, Free and Guided Propagation. Springer-Verlag, Berlin, (2000). 2

55. Ryu, J. and Shashank, P. and Kenji, U. and Kim, H.: Magnetoelectric effect in composites of magnetostrictive and piezoelectric materials. J. Electroceram. 8, 107-119 (2002). 2 
56. Sharma, J. N. and Kumar, V. and Chand, D.: Reflection of generalized thermoelastic waves from boundary of a halfspace. J. Thermal Stresses 26, 925-942 (2003). 3, 15

57. Sharma, J. N. and Walia, V. and Gupta, S. K.: Reflection of piezothermoelastic waves from the charge and stress free boundary of a transversely isotropic half space. Int. J. Eng. Sci. 46(2), 131-146 (2008). 3, 7

58. Sharma, J. N. and Walia, V. and Gupta, S. K.: Effect of rotation and thermal relaxation on Rayleigh waves in piezothermoelastic half space. Int. J. Mech. Sci. 50, 433-444 (2008). 5

59. Lord, H. W. and Shulman, Y.: A generalized theory of thermoelasticity. J. Mech. Phys. Solids 15, 299-309 (1967). 2, 5

60. Singh, B.: On the theory of generalized thermoelasticity for piezoelectric materials. Applied Mathematics and Computation 171(1), 398-405 (2005). 3, 7

61. Singh, B.: Wave propagation in a prestressed piezoelectric half-space. Acta Mechanica 211(3-4), 337-344 (2010). 3

62. Singh, S. S.: Transverse wave at a plane interface in thermo-elastic materials with voids. Meccanica 48(3), 617-630 (2013). 2, 3

63. Song, Y. Q. and Bai, J. T. and Ren, Z. Y.: Study on the reflection of photothermal waves in a semi-conducting medium under generalized thermoelastic theory. Acta Mech. 223, 1545-1557 (2012). 2

64. Srinivasan, G. and Laletsin, V.M.: Giant magnetoelectric effects in layered composites of nicked zinc ferrite and lead zirconate titanate. Solid State Commun. 124, 373-378 (2002). 2

65. Sciarra, G. and dell'Isola, F. and Ianiro, N. and Madeo, A.: A variational deduction of second gradient poroelasticity part I: General theory. Journal of Mechanics of Materials and Structures 3(3), 507-526 (2008). 3

66. Spencer, A. J. M.: The formulation of constitutive equation for anisotropic solids. Mechanical behavior of anisotropic solids, edited by J. P. Boehler, Martinus Nijhoff Publishers, The Hague, 2-26 (1982). 5

67. Tomar, S. K. and Khurana, A.: Elastic waves in an electro-microelastic solid. Int. J. Solids and Structures 45, 278-302 (2008). 3

68. Van Suchtelen, J.: Product properties: a new application of composite materials, Philips. Res. Rep. 27, 28-37 (1972). 2

69. Vidoli, S. and dell'Isola, F.: Vibration control in plates by uniformly distributed PZT actuators interconnected via electric networks. European Journal of Mechanics, A/Solids 20(3), 435-456 (2001). 2

70. Wu, L.: Piezoelectric thin film: Fabrication, Properties and Applications. Proceeding of The Second Symposium on Piezoelectricity, Acoustic Waves, and Device Applications 5, (2007). 2

71. Yang, J.: The mechanics of piezoelectric structures. World Scientific Publishing Co., Singapore (2008). 2

72. Yang, J.: An introduction to the theory of piezoelectricity. Springer Science, Boston U.S.A. (2005). 2

73. Yuan, X. and Kuang, Z.: Waves in Pyroelectrics. Journal of Thermal Stresses 31(12), 1190-1211 (2008). DOI: 10.1080/01495730802508046. 6

74. Ye, Z.G.: Handbook of dielectric, piezoelectric and ferroelectric materials Synthesis, properties and applications. Woodhead Publishing Limited, CRC Press, New York (2008). 2

75. Yuan, X. and Zhu, Z. H.: Reflection and refraction of plane waves at interface between two piezoelectric media. Acta Mech. 223, 2509-2521 (2012). 